\section{A New Type of Pictorial Database: The Bicolor Affective Silhouettes and Shapes (BASS)}

$$
\text { Claudia Kawai }^{1 *} \text {, Gáspár Lukács }{ }^{1} \text {, and Ulrich Ansorge }{ }^{1,2,3}
$$

\section{${ }^{1}$ Department of Cognition, Emotion, and Methods in Psychology, University of Vienna}

${ }^{2}$ Vienna Cognitive Science Hub, University of Vienna

${ }^{3}$ Research Platform Mediatized Lifeworlds, University of Vienna

\section{Claudia Kawai (D) https://orcid.org/0000-0001-5149-6921}

Gáspár Lukács (iD https://orcid.org/0000-0001-9401-4830

Ulrich Ansorge (iD https://orcid.org/0000-0002-2421-9942

Correspondence concerning this article should be addressed to Claudia Kawai, Faculty of Psychology, University of Vienna, Liebiggasse 5, A-1010 Vienna, Austria. E-mail: claudia.kawai@univie.ac.at 
Abstract

19 We introduce the Bicolor Affective Silhouettes and Shapes (BASS): a set of 583 normed black20 and-white silhouette images that is freely available via osf.io/anej6. Valence and arousal ratings 21 were obtained for each image from US residents as a Western population $(n=777)$ and Chinese 22 residents as an Asian population $(n=869)$. Importantly, the ratings demonstrate that, 23 notwithstanding their visual simplicity, the images represent a wide range of affective content 24 (from very negative to very positive, and from very calm to very intense). In addition, speaking 25 to their cultural neutrality, the valence ratings correlated very highly between US and Chinese 26 ratings. Arousal ratings were less consistent between the two samples, with larger discrepancies 27 in the older age groups inviting further investigation. Due to their simplistic and abstract nature, 28 our silhouette images may be useful for intercultural studies, color and shape perception research, and online stimulus presentation in particular. We demonstrate the versatility of the BASS by an example online experiment. research 


\section{A New Type of Pictorial Database: The Bicolor Affective Silhouettes and Shapes (BASS)}

Images are an excellent medium to convey information, simple or complex. They serve as stimuli in a wide range of research areas, such as emotion, attention, or aesthetics research, among others (e.g., Bradley \& Lang, 2007; Huston et al., 2015; Lindsay, 2020). In the present article, we introduce an open access database of normed Bicolor Affective Silhouettes and Shapes (BASS). ${ }^{1}$ Each of the BASS images consists of $300 \times 300$ black and white pixels, providing a computationally economical and visually uniform layout. ${ }^{2}$

New freely available normed images enrich research by providing greater diversity of choices for research, including potential replications of findings with different kinds of images to ensure generalizability. Additionally, participants may get habituated to the images in existing databases, meaning they may process them differently on repeated exposures (Baker et al., 2010; Foa \& Kozak, 1986; Ramaswami, 2014). The present BASS database may be used, for instance, for conceptual replications and novel research in such diverse areas as space-valence congruence effects (e.g., Meier \& Robinson, 2004), priming of evaluations (e.g., Fazio, 2001), affective priming (e.g., Hermans et al., 2001), or emotional facilitation (e.g., Schupp et al., 2003).

${ }^{1}$ Some of the BASS images are not silhouettes in the strictest sense, but rather silhouettelike shapes because they also illustrate hollow parts or outlines (e.g., the edges and dots on dice). Nonetheless, for simplicity, in the present article, we refer to all the BASS images as "silhouettes."

${ }^{2}$ Several databases of shapes or objects do exist, like MultiPic (Duñabeitia et al., 2018), ALOI (Geusebroek et al., 2005), the sets by Nishimoto et al. (2012), by Brady et al. (2013), or by Snodgrass and Vanderwart (Rossion \& Pourtois, 2004; Snodgrass \& Vanderwart, 1980). However, these were not created (let alone verified or normed) with the intention of eliciting affect: The majority of these images depict everyday objects or animals. With a few exceptions, like dangerous versus cute animals, or weapons, it is unlikely that they would elicit strong affective responses or emotions. In the OASIS, for example, people and scene pictures were rated as more emotional than objects (Kurdi et al., 2017, p. 463), which suggests that these databases are not ideal for emotion research purposes. 
More importantly, the BASS also has very specific advantages compared to other available stimulus sets: Silhouette images allow for the study of meaning-related processing without many of the confounds that are present in words and pictures. Whereas words carry inherent differences, such as their length and their phonological transparency (or orthographic depth, see Aro \& Wimmer, 2003; Frost et al., 1987; Schmalz et al., 2016) for which one would have to control, these confounding differences are absent in silhouettes. Likewise, whereas pictures differ from one another in terms of color heterogeneity, depth cues, spatial perspective, or feature complexity, silhouettes are more uniform and less complex, and can therefore be more easily equated for these factors, without corrupting their meaning entirely.

The main purpose of the present research article is to demonstrate that the BASS images evoke a very wide range of affective representations on the side of the participants - despite the images' relative simplicity. We demonstrate this primarily by collecting valence and arousal ratings in a large and nationally representative US (Western) sample. We provide further evidence from analogous ratings from an extensive (Eastern) sample from Mainland China and from demonstrating characteristic valence-color congruence effects in an example response time experiment using BASS images (in an Austrian sample).

This methodological approach validates several important characteristics of the BASS images, relevant to but often not investigated in image sets used in psychological research. First, typically, normative ratings of affective picture databases like the Geneva Affective Picture Database (GAPED), the Open Affective Standardized Image Set (OASIS), the International Affective Picture System (IAPS), or the Nencki Affective Picture System (NAPS) (Dan-Glauser \& Scherer, 2011; Kurdi et al., 2017; Lang et al., 1997; Marchewka et al., 2014, respectively) are largely based on mono-cultural samples, with ratings predominantly collected in Western 
71 populations only. Moreover, within Western populations, samples were often restricted to student participants. ${ }^{3}$ Yet, ideally, behavioral image or stimulus sets should be applicable in different cultures in order to allow investigation of universal psychological phenomena and topics. The IAPS, for example, has been generally found to be valid among Western cultures (Deák et al., 2010; Grühn \& Scheibe, 2008; Verschuere et al., 2001, to name a few), but less so among Asian cultures (Gong \& Wang, 2016; Huang et al., 2015). Therefore, from the start, for the current BASS images, we intended to collect normative rating data from at least two large, representative samples of different cultural origin: one Western (US) and one Eastern (Mainland China) sample. Silhouette images theoretically carry potential for more culturally neutral affective representations than photographic images, due to the latter's culture-specific details (such as depictions of regionally different vegetation, landscapes, food, people, architecture, traffic etc.), which are often subtle and difficult to control for. We tested this conceptual notion by directly comparing the consistency of the ratings from our Western and Asian samples. Second, the bicolor silhouettes tackle one particular virulent problem posed by online studies: accurate color rendition of heterogeneous colors on different physical displays. By implication of their high realism, color photos of natural scenes carry substantial color heterogeneity and, in fact, their realism relies in part on this color heterogeneity (cf. Hansen \& Gegenfurtner, 2009). It has indeed been shown that differences in the display of photographs, such as varying brightness or resolution, can substantially affect the evaluation of emotional content (e.g., Lakens et al., 2013; Mould et al., 2012). However, the huge variety of physical

${ }^{3}$ IAPS: US undergraduate students, OASIS: more diverse but predominantly white US participants, GAPED: South/West-European psychology students, NAPS: Polish and other mostly European students. 
devices used to display images (e.g., smartphones, tablets, and laptops by different manufacturers) plus an increased variance in idiosyncratic monitor settings and lighting conditions on the side of the viewer compromise accurate color rendition, especially in online studies (Anwyl-Irvine et al., 2020) - a problem which increases with the complexity of the image's color palette. In contrast, the black-and-white silhouettes of the BASS database were already created and selected (from existing silhouettes) under the premise of having to convey their affective meanings largely by their contours alone and less by their exact color rendition. In this way, the BASS images reduce the heterogeneity of the necessary color palette for their rendition and the problems associated with this color heterogeneity. We demonstrated this by collecting participants' affective ratings using the BASS images in inverse colors (pilot study). However, this is not to say that colors could not be manipulated and used to study their effects (e.g., on affective ratings) in studies with BASS images. For photographs, manipulations of color rendition can easily corrupt image meaning altogether (cf. Oliva \& Schyns, 2000; Mould et al., 2012). Such manipulation requires complicated technical procedures (e.g., Orzan et al., 2007), and, even more fundamental, one can hardly be sure what manipulation of colors in a photography affects emotional image content in what manner: Setting up a proper balance of hue, saturation, and brightness is difficult enough when calibrating the display of a single color (Wilms \& Oberfeld, 2018), let alone for a photograph with thousands of interacting colors. In contrast, the BASS images were created with the intention of keeping an unambiguous meaning by way of their contours, even if presented in varying colors. Thus, BASS images offer an extremely easy way to manipulate and study or control color: They can be converted effortlessly 
112 to any other two colors ${ }^{4}$ - and, as long as the two colors are discernible from each other, the

113 content remains unambiguous. Below, we also demonstrate how such color manipulations of the

114 BASS images could be used in research by investigating known color-valence congruence

115 effects using variously colored BASS images for an example experiment.

116 Third, large photographic images are generally not optimal for fast decision tasks based

117 on their diverse visual characteristics and complex semantics: Various depicted details and their

118 many subtle visual properties might affect each participant very differently, influencing

119 processing time and potentially confounding results (especially where small effects are expected

120 and processing-time differences in fractions of seconds can affect outcomes). In contrast, the

121 contents of the less ambiguous (few or single) objects in each BASS image is easy to grasp

122 quickly and better suited for a task involving fast decisions, unaffected by visual or semantic

123 noise. In addition, their simplistic and abstract nature makes BASS silhouettes more resistant

124 than photographs to incidental but potentially meaning-corrupting influences such as display size

125 or viewing distance (Anwyl-Irvine et al., 2020). ${ }^{5}$ Big file sizes of photographic images used in

126 experiments typically pose technical problems too, especially in connection with online research,

127 which is becoming more and more important in psychological science. For one, common

128 browsers are not optimized for precisely timing image presentation, and physical display times

129 can be strongly affected by loading large size images (e.g., Garaizar \& Reips, 2019). For another,

130 some participants might be reluctant (or even unable, where infrastructure is weak) to download

131 many large files. It is not unusual for an experiment to require several hundreds of different

${ }^{4}$ An R script for easy instant conversion of any number of images to any chosen color is available via https://github.com/gaspar1/BASS.

5 The contents of most BASS images can be easily recognized even when they are strongly blurred or scaled to icon size. 
132 stimuli (especially when each stimulus is presented repeatedly in different forms, e.g., varying 133 hue, etc.; e.g., Huang et al., 2015; Kawai et al., 2020; Oliva \& Schyns, 2000). Calculating with 134 the average file size of IAPS images $(262 \mathrm{~KB}$; cf. averages of $92 \mathrm{~KB}$ for OASIS and $789 \mathrm{~KB}$ for

135 GAPED), just 100 images take $26 \mathrm{MB}$ - already a notable hurdle for potential participants.

136 Participant dropout due to inadequate internet speed could even introduce confounds via

137 selective attrition (Zhou \& Fishbach, 2016). With an extremely economical average file size of $1382.2 \mathrm{~KB}$ (max. 6.0 KB), the BASS images minimize the issues of display timing and download.

139 Our follow-up experiment on color-valence congruence effects also serves to demonstrate these 140 usability advantages in fast decision tasks online.

141 Fourth, silhouettes, contours, and shapes themselves constitute a field of scientific

142 interest (e.g., Bar, 2007; Biederman, 1987; Marr, 1982), and the affective qualities associated

143 with geometric contour properties may be of interest to future researchers (e.g., Leder et al.,

144 2011), although they have not been a major focus of research on contours so far. This could be

145 particularly interesting when comparing human object recognition with artificial object

146 recognition (e.g., Rajalingham et al., 2018): While objects as simple as silhouettes may be

147 processed by machines similarly to humans, affective responses are (currently) only evoked in

148 humans.

\section{Creating the BASS}

Before carrying out the large-scale ratings for the BASS, we assessed several issues in a

152 pilot study ( $n=180$, students of the University of Vienna). Detailed information on the pilot

153 study can be found in the online supplementary material available on osf.io/anej6. Firstly,

154 researchers suspected, but never tested, possible contamination of affective ratings when

155 participants had to rate valence and arousal in one experiment (Kurdi et al., 2017), since prior 
studies showed that question order in surveys can influence judgements (Lau et al., 1990; Wilcox \& Wlezien, 1993). We put this assumption to the test and split participants into two groups: one rating only valence (or only arousal) and another rating images on both valence and arousal. Results indeed confirmed contamination in affective ratings when two affective ratings were required in the same experiment. Thus, we decided to use only one rating measure (either valence or arousal) per participant in the main study. In the pilot study, we simultaneously tested for the relative-color independence of the silhouette ratings by studying possible effects of colorinversion of silhouettes (black-on-white vs. white-on-black) on ratings. High positive correlations between the (original) black-on-white images and the inverted white-on-black images suggested that there is no apparent preferred color mode for ratings of our silhouettes (clarity ratings: $r[618]=.866,95 \%$ CI $[.845, .885]$; valence: $r[618]=.918,95 \%$ CI $[.905, .930]$; arousal: $r[618]=.719,95 \%$ CI $[.678, .755])$. The ratings from the pilot study, given by Austrian psychology students, also helped us to decide on a reasonable exclusion threshold for intrarater correlation, which we subsequently used as an individual reliability measure for data quality control in the main study. Since intrarater correlation turned out to be lower in the arousal rating than in the valence rating condition, we determined different exclusion thresholds depending on the rating condition (see Data Exclusion).

Clarity ratings provided by the first as well as a second online prestudy $(n=50$, Chinese participants) helped to identify unclear or ambiguous images in the original set of 620 . After excluding those with the lowest clarity ratings, the subset that was used in the large-scale ratings in the US and China comprised a total of 583 silhouettes. 
177 Method

\section{Participants}

179 For the US rating, a total of 806 participants was recruited via Prolific (www.prolific.co).

180 The study was divided into two parts: one sub-study comprised the valence rating $(n=402)$, the

181 other the arousal rating $(n=404)$ task. The sample was collected as a "representative sample for

182 the United States of America" (sex, age, ethnicity, according to Simplified US Census; excluding

183 people under the age of 18 ) and participants received $£ 2.17$ upon study completion.

184 The experiment had to be completed at a desktop computer. Participants could read all

185 relevant information on the welcome page and consented to participate by clicking the consent

186 button at the bottom.

After exclusion (see Data Exclusion), valid data from 777 participants remained (arousal rating: 386; valence rating: 391), out of which 402 participants were female, 375 were male. Ages ranged from 18 to 78 years, with a mean age of 45.19 years $(S D=16.09) .{ }^{6}$ Completion took an average of $17 \mathrm{~min}(S D=4 \mathrm{~min}$, median $=15 \mathrm{~min})$.

For the Chinese sample, the recruitment (and compensation) of online participants in

192 China was managed by Dynata. In the supplementary materials, we provide more information on

193 Dynata's incentive and compensation system. Chinese participants were asked to provide

194 demographic data after consenting to the study. We only allowed participation for those who

195 indicated (1) their age being between 18 and 95 years, (2) Mainland China as their country of

196 residence, and (3) any gender information. A total of 1,341 registered participants submitted their

197 rating data, of which 472 had to be excluded due to poor data quality (for more details see Data

${ }^{6}$ For two US participants, we do not know their definite ages, only which age group they belong to. Mean age and $S D$ are calculated without these two participants. 
198 Exclusion). We aimed for a representative sample (sex; age, excluding people under the age of

19918 years) for the People's Republic of China, but since our study required PC and internet

200 access, relatively younger age groups are over-represented.

201 After exclusion, valid data from 869 participants remained (arousal rating: 455; valence

202 rating: 414), out of which 419 participants were female and 450 were male. Ages ranged from 18

203 to 81 years, with a mean age of 34.5 years $(S D=9.4)$. Completion took on average $25 \mathrm{~min}(S D=$

204116 min, median $=14 \mathrm{~min})^{7}$

205 An overview of the demographic data from all included participants (US and China) is

206 provided in Table A1 (Appendix A).

207

208

\section{Materials}

The majority of the silhouettes were acquired using Google Images (images.google.com)

210 under the creative commons license, by searching either directly for silhouettes or for

211 photographic pictures that we edited into black-and-white silhouettes using GNU Image

212 Manipulation Program (GIMP) and R (R Core Team, 2020).

213 We restricted our search to images labelled "available for reuse with modification." Most

214 silhouettes are from pixabay.com $(n=444)$, cleanpng.com $(n=33)$, and svgsilh.com $(n=29)$.

215 Eighteen additional silhouettes were created for this project by a professional illustrator. We

216 collected a wide range of images, depicting humans, animals, objects, and scenes, which we

217 indicated by a category column in the database for easier stimulus selection. Special focus was

${ }^{7}$ Duration ranged from $10 \mathrm{~min}$ to 53 hours. The latter was an extreme outlier that is no doubt due to a measurement error: For example, the participant may have opened the link to the task but only began completing it after a very long pause. 
218 put on collecting images of a wide range of valence (positive, negative, and neutral) as well as 219 arousal (low, medium, and high) levels. Every image was scaled and/or cropped to a size of 300 $220 \times 300$ pixels. All images were checked (or converted) to contain only fully black (RGB: $0,0,0)$ 221 and fully white (RGB: 255, 255, 255) pixels.

\section{Procedure}

Online Rating Study - US. After giving their informed consent, participants saw the instruction page with an explanation of the terms valence and/or arousal (see Figures A1 and A2 in Appendix A), depending on the participants' rating condition, and three example images (selected based on the pilot studies as average in both arousal and valence; none of these images were used in the subsequent task for the given participant). The experiment started as soon as the "Start" button on the bottom of the instruction page was pressed. Each black-and-white silhouette stimulus $(300 \times 300 \mathrm{px})$ was presented on a grey background (RGB: $128,128,128)$ for $2 \mathrm{~s}$. When this time had elapsed, the image disappeared and was replaced with the rating scale, a line ranging from "very low" on the left to "very high" on the right, with nine equally spaced tick marks (see Figure A3). Participants could enter their ratings by clicking on the scale(s) and submit their rating by pressing a button that appeared below, or they could skip the rating. After confirming their choice, the next image appeared on the screen, and so on. A total of 145-146 silhouettes were presented to each participant. Additionally, we incorporated two attention checks. Hyperlinks to the original experimental websites are available via osf.io/anej6.

Attention Checks. After rating every stimulus in the list, a black-and-white image was shown after which the participant had to indicate what was depicted on it out of a list of answers (this was the same item for all participants, a clearly discernible black-and-white image of a car). 
240 In addition, we assessed participants' attention via intrarater reliability. Following the first

241 attention check, the participant was presented with five of their most highly and five of their

242 most lowly rated images (in randomized order). We calculated Spearman's correlation

243 coefficient between the first and second ratings for these 10 items.

244 Online Rating Study - China. The procedure of the experiment was identical to that

245 used for the US sample, with the exception that Chinese participants were asked to provide

246 demographic data (gender, age, country of residence) after giving consent to the study. ${ }^{8}$ The

247 experimental website including the informed consent and instruction pages was translated into

248 Mandarin Chinese by a native speaker (a psychologist) and independently double-checked by

249 another native speaker (a linguist).

Data Exclusion

Participants were excluded according to the following criteria: (1) the rate of skipped

252 answers was above $25 \%$; (2) no intrarater correlation could be calculated ${ }^{9}$ (3) for arousal ratings,

253 a failed Attention Check 1 and an intrarater correlation of less than .77 or otherwise a passed

254 Attention Check 1 but an intrarater correlation of less than .67; (4) for valence ratings, a failed

255 Attention Check 1 and an intrarater correlation of less than .80; or otherwise a passed Attention

256 Check 1 but an intrarater correlation of less than $.70 .{ }^{10}$ In addition, in the Chinese rating, data

${ }^{8}$ The demographic data for people who participated in the representative US rating was provided by Prolific.

${ }^{9}$ This can either happen when the repetition items needed to calculate the correlation were skipped or when the same rating, for example, "5", was given on every trial.

${ }^{10}$ Exclusion criteria 3-4 were empirically determined by pilot testing in the pilot study, which is described in more detail in the supplementary online material. 
from participants was excluded if they did not provide a personal identification code (generated by Dynata). Surprisingly, exclusion rates for the China data $(472 / 1,341=35.2 \%)$ were much larger than that for the US data $(29 / 806=3.6 \%)$. This may have happened for various reasons, but a major difference is that Prolific (US data) specializes in collecting data for scientific purposes, while Dynata serves mainly as a provider of market research data.

\section{Results}

Each image received between 91 and 119 ratings. For each country, we computed mean valence and mean arousal ratings per image.

\section{US Rating}

Reliability. For valence and arousal ratings, separately, we generated 1,000 random split halves of our sample and calculated the Spearman-Brown reliability coefficient of the mean ratings. The mean of the correlation coefficients in the valence dimension was $\bar{R}_{v a l}=.986(S D=$ $5.51 \times 10^{-5}$, range: $R_{\min }=.985$ and $\left.R_{\max }=.986\right)$, and in the arousal dimension $\bar{R}_{\text {aro }}=.921(\mathrm{SD}$ $=3.50 \times 10^{-4}$, range: $R_{\min }=.920$ and $\left.R_{\max }=.929\right)$, demonstrating extremely high reliability in both affective dimensions.

Relationship between Valence and Arousal. Mean valence ratings from the US participants lie between 1.11 and $8.35(M=5.64, S D=1.76)$; mean arousal ratings lie between 2.51 and $7.23(M=5.00, S D=1.02)$. The correlation between valence and arousal ratings was significant, with $r(581)=-.323,95 \%$ CI $[-.394,-.248], p<.001, B F_{10}=5.76 \times 10^{12}$. However, the Pearson correlation coefficient shows a relatively weak linear relationship and a look at the 
280 distributions of the US mean ratings, as shown in Figure 1, suggests a U-shaped relationship

281 between valence and arousal in the BASS images, where arousal dips for neutral images but then 282 increases with rising valence. ${ }^{11}$

11 The same U-shaped relationship was found in the IAPS and OASIS. Our observed valence-arousal correlation is very similar to that of the IAPS, which shows $r(1,192)=-.289$, $95 \%$ CI $[-.340,-.236], p<.001, B F_{10}=2.16 \times 10^{21}$, while the correlation in the OASIS was not significant, with $r(898)=-.058,95 \%$ CI [-.123, .007], $p=.082, B F_{01}=2.84$. 


\section{Figure 1}

284 Mean Ratings (95\% CI Error Bars) per Image for Valence and Arousal from US Participants. To

285 Illustrate the Tendency of the Relation, the Blue Line Depicts Locally Weighted Regression 286 (LOWESS).

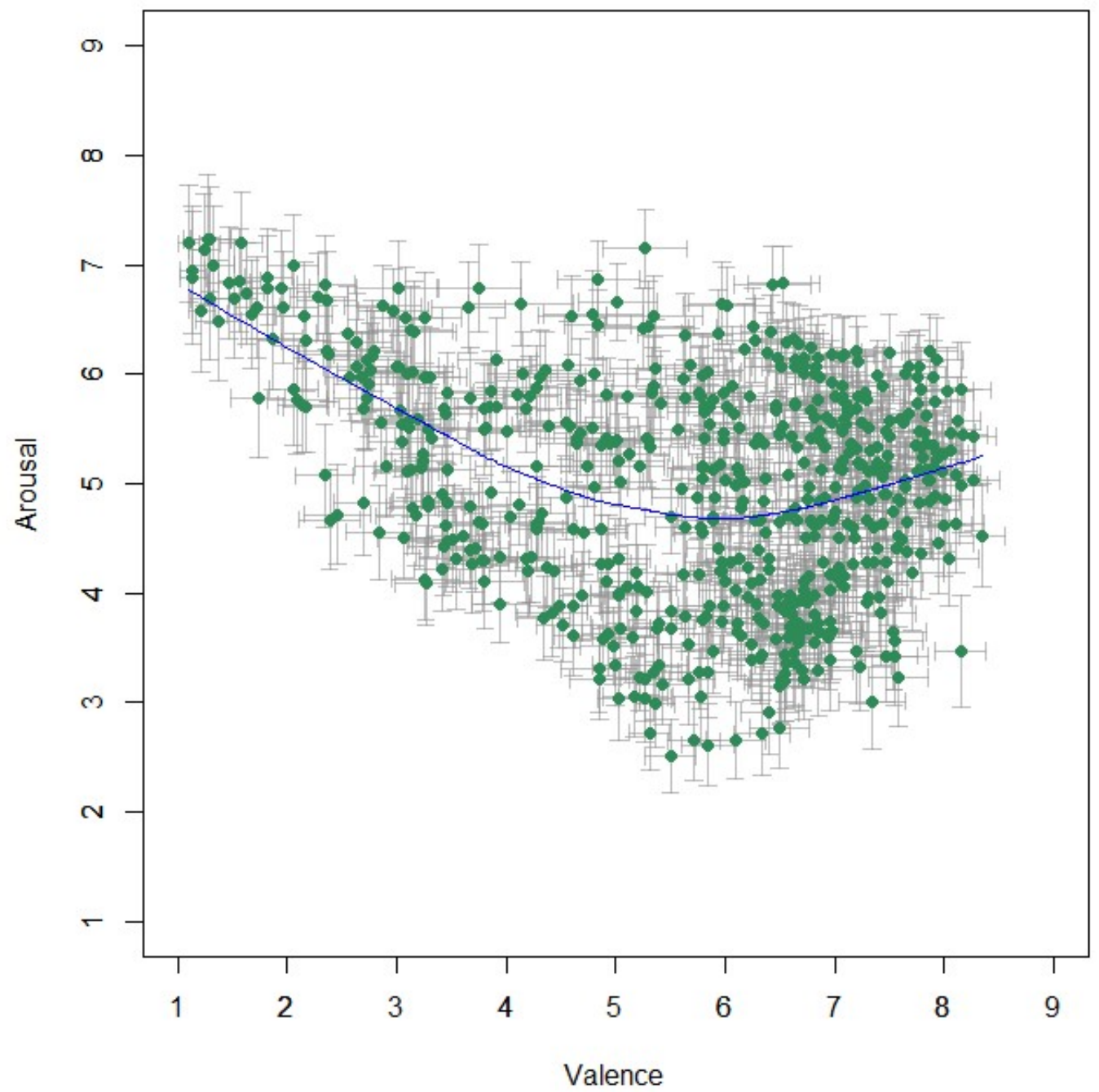


Effects of Demographic Variables. Prior research has identified differences in emotion processing based on factors like gender (Bradley et al., 2001; Proverbio et al., 2009; Sabatinelli et al., 2004; Wrase et al., 2003) and age (Bradley \& Lang, 2007; Grühn \& Scheibe, 2008; Pôrto et al., 2010). To investigate potential gender-related differences in our affective ratings, we calculated the individual means for valence and arousal ratings for male and female US participants.

The correlation between the mean ratings made by male and female participants was very high, for both valence, $r(581)=.972,95 \%$ CI $[.967, .976], p<.001, B F_{10}>10^{200}$, and arousal, $r(581)=.877,95 \% \mathrm{CI}[.857, .895], p<.001, B F_{10}=2.31 \times 10^{182}$. Mean valence and arousal ratings (per item) for male and female participants are displayed in Figure 2. For age-wise comparison, we performed a median split for the demographic variable age on both of our US subsamples (valence, arousal) and correlated the respective mean ratings of the halves. For the valence rating group (median age $=46$ years), correlation between the younger $(<46$ years) and the older $(\geq 46$ years) age group was very high, with $r(581)=.976$, $95 \%$ CI $[.972, .979], p<.001, B F_{10}>10^{200}$. The same was true for the arousal rating group 303 (median age $=44.5$ years), with a correlation between the younger $(<45$ years) and the older $(\geq$ 30445 years) age group of $r(581)=.906,95 \%$ CI $[.891, .920], p<.001, B F_{10}>10^{200}$. Finally, Figure 3053 shows that the valence-arousal pattern is very similar for all age groups (divided into five 306 brackets following Prolific's age stratification, see Table A1). 
BASS

307 Figure 2

308 Mean Valence by Mean Arousal Ratings per Picture for Male versus Female US Participants

309 (LOWESS Line in Green and Blue, Respectively).

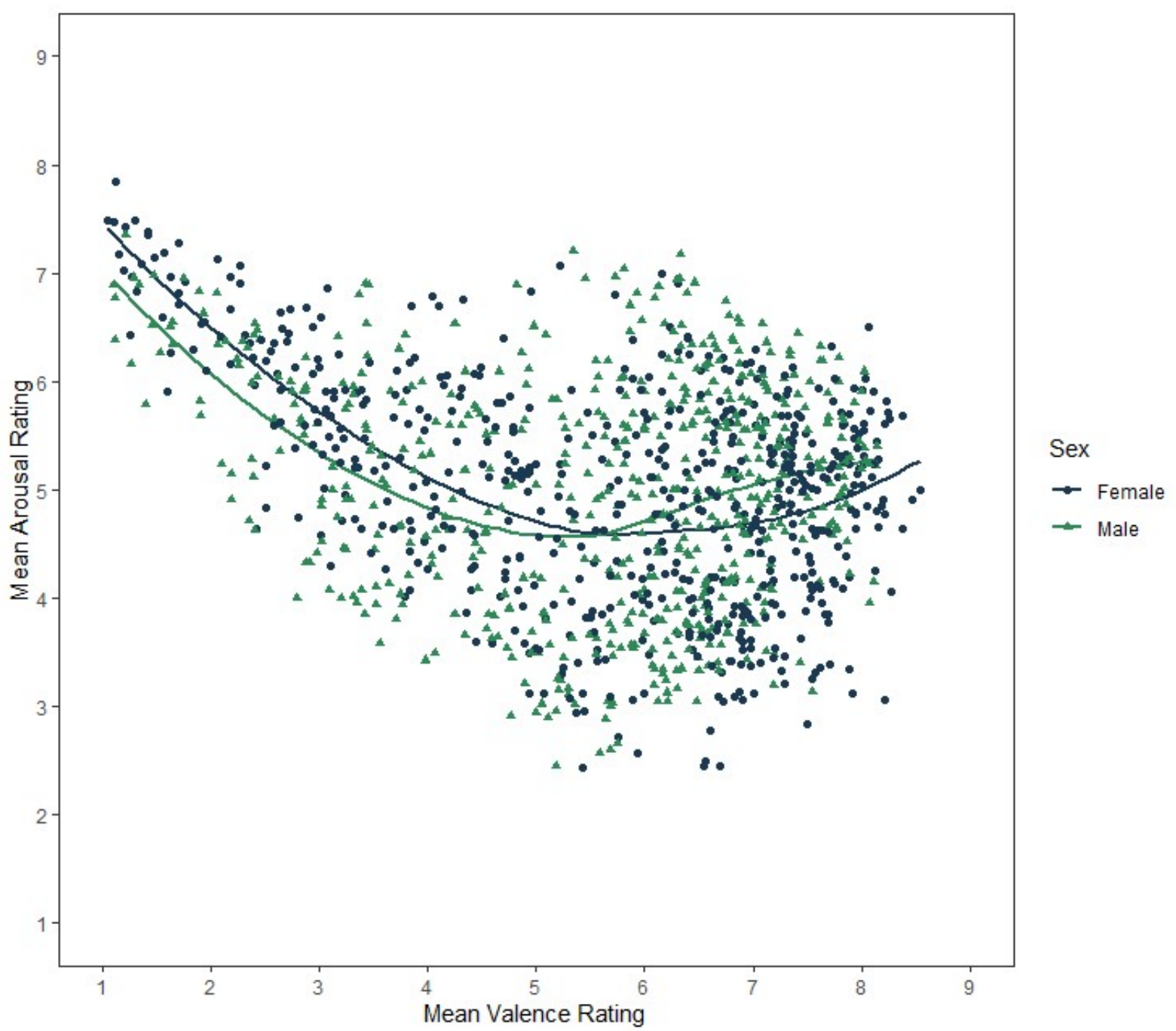


BASS

315 Figure 3

316 Mean Valence by Mean Arousal Ratings per Picture by Age Group from US Participants, with

317 Corresponding LOWESS Lines.

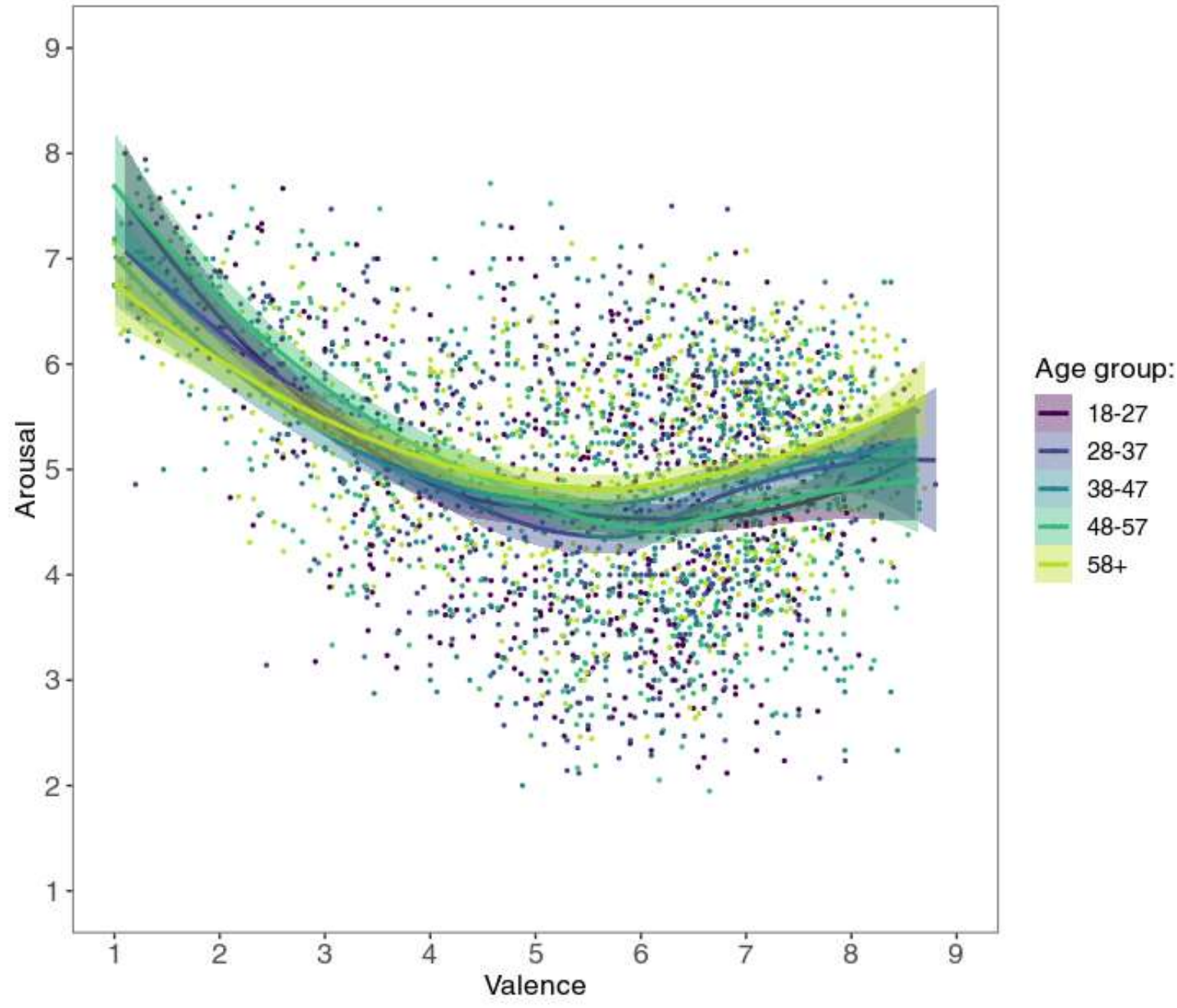

318 


\section{Chinese Rating}

Reliability. The mean of the correlation coefficients in the valence dimension was $\bar{R}_{\text {val }}$

$322=.980\left(S D=7.64 \times 10^{-5}\right.$, range: $R_{\min }=.979$ and $\left.R_{\max }=.980\right)$, and in the arousal dimension $\bar{R}_{\text {aro }}=.921\left(S D=2.87 \times 10^{-4}\right.$, range: $R_{\min }=.917$ and $\left.R_{\max }=.923\right)$. Interrater reliability for both affective dimensions was again extremely high and almost identical to the US sample.

Relationship between Valence and Arousal. Mean valence ratings from the Chinese participants lie between 1.47 and $7.84(M=5.46, S D=1.37)$; mean arousal ratings lie between 3.65 and $6.75(M=5.31, S D=0.64)$. Unexpectedly, and different from the results in the US sample, mean arousal and mean valence ratings showed a strong positive correlation, with $r(581)$

$329=.805,95 \%$ CI $[.774, .832], p<.001, B F_{10}=2.33 \times 10^{129}$. The distributions of the Chinese mean

330 valence and arousal ratings are shown in Figure 4, which illustrates that negative images

331 received relatively low arousal ratings while positive images received relatively high arousal

332 ratings (as opposed to the US ratings, where arousal ratings were distributed fairly evenly

333 between positive and negative images). 
BASS

335 Figure 4

336 Mean Ratings (95\% CI Error Bars) per Image for Valence and Arousal from Chinese

337 Participants with the LOWESS Line in Blue.

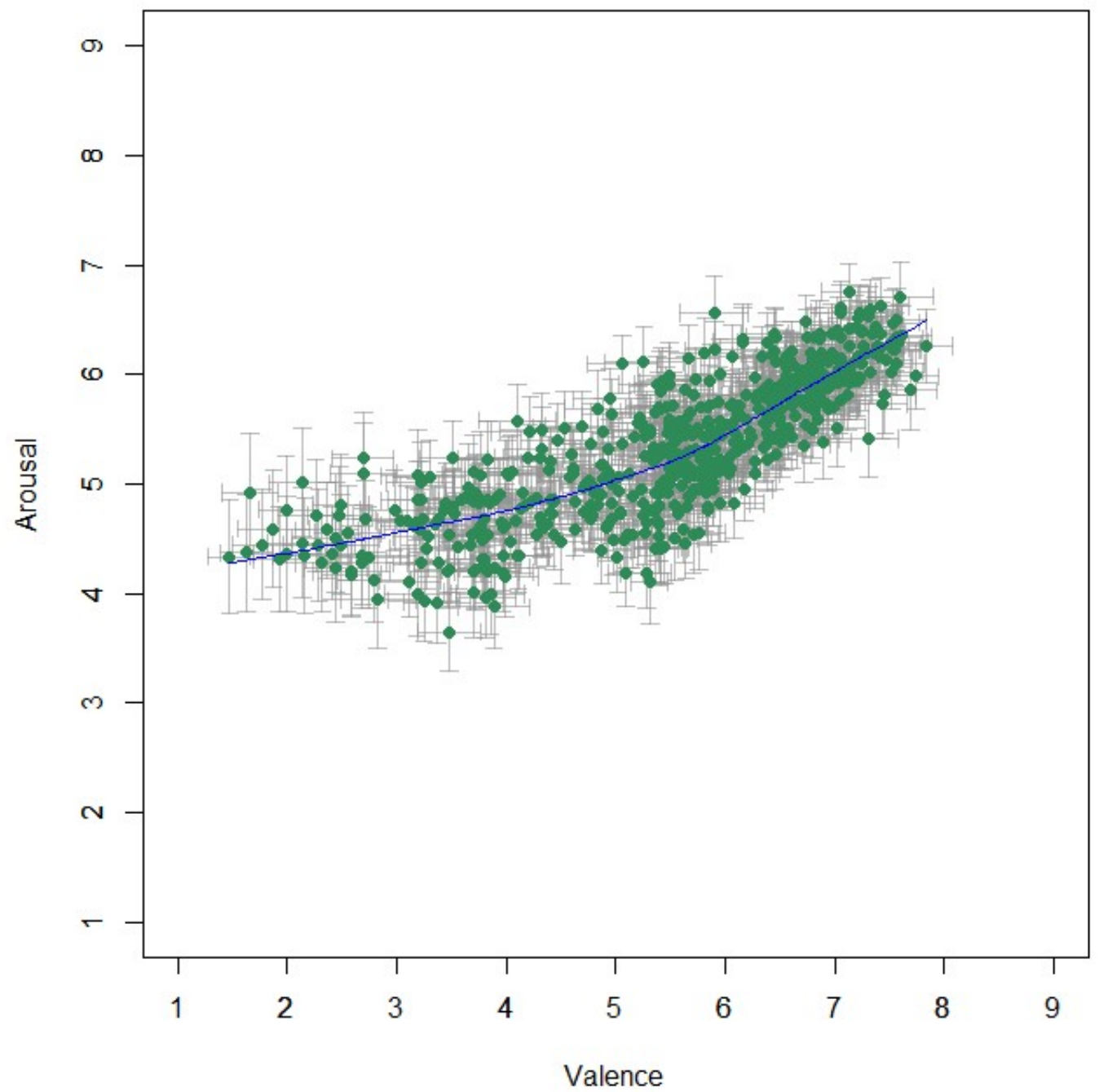


Effects of Demographic Variables. Similarly to the US sample, mean ratings between

340 Chinese male and female participants were highly correlated for valence, $r(581)=.979,95 \%$ CI

$341[.975, .982], p<.001, B F_{10}>10^{200}$, and arousal, $r(581)=.856,95 \% \mathrm{CI}[.832, .876], p<.001$,

$342 B F_{10}=4.11 \times 10^{163}$. Mean valence and arousal ratings (per item) for male and female

343 participants are displayed in Figure 5.

344 For age-wise comparison, we again performed a median split for age on both Chinese

345 subsamples (valence, arousal). For the valence rating group (median age $=32$ years), correlation

346 between the younger $(<32$ years $)$ and the older ( $\geq 32$ years) age group was extremely high, with $347 r(581)=.974,95 \%$ CI $[.970, .978], p<.001, B F_{10}>10^{200}$. For the arousal rating group (median

348 age $=33$ years $)$, the agreement between the younger $(<33$ years $)$ and the older $(\geq 33$ years $)$ age

349 group was still very high, although somewhat lower, $r(581)=.785,95 \%$ CI $[.752, .814], p$

$350<.001, B F_{10}=3.41 \times 10^{118}$. Note that the median age was much lower in the Chinese compared

351 to the US sample: The Chinese sample was split in the early 30 s while the US sample was split

352 in the mid-40s.

353 As for the US sample, five age brackets were created - however, the ratings by Chinese

354 age group " $58+$ " are hardly reliable since this sample consists of a mere 14 participants -

355 therefore they are omitted from the present figures. (They are nonetheless largely in line with the

356 rest of the data; see supplementary figures at osf.io/anej6.) Most interestingly, the different age

357 groups show somewhat different patterns (see Figure 6), with ratings from younger age groups,

358 especially the youngest (ages 18-27), tending to resemble more to the US sample and the U-

359 shape often observed in Western samples. 
BASS

$362 \quad$ Figure 5

363 Mean Valence by Mean Arousal Ratings per Picture for Male versus Female Chinese

364 Participants (LOWESS Line in Green and Blue, Respectively).

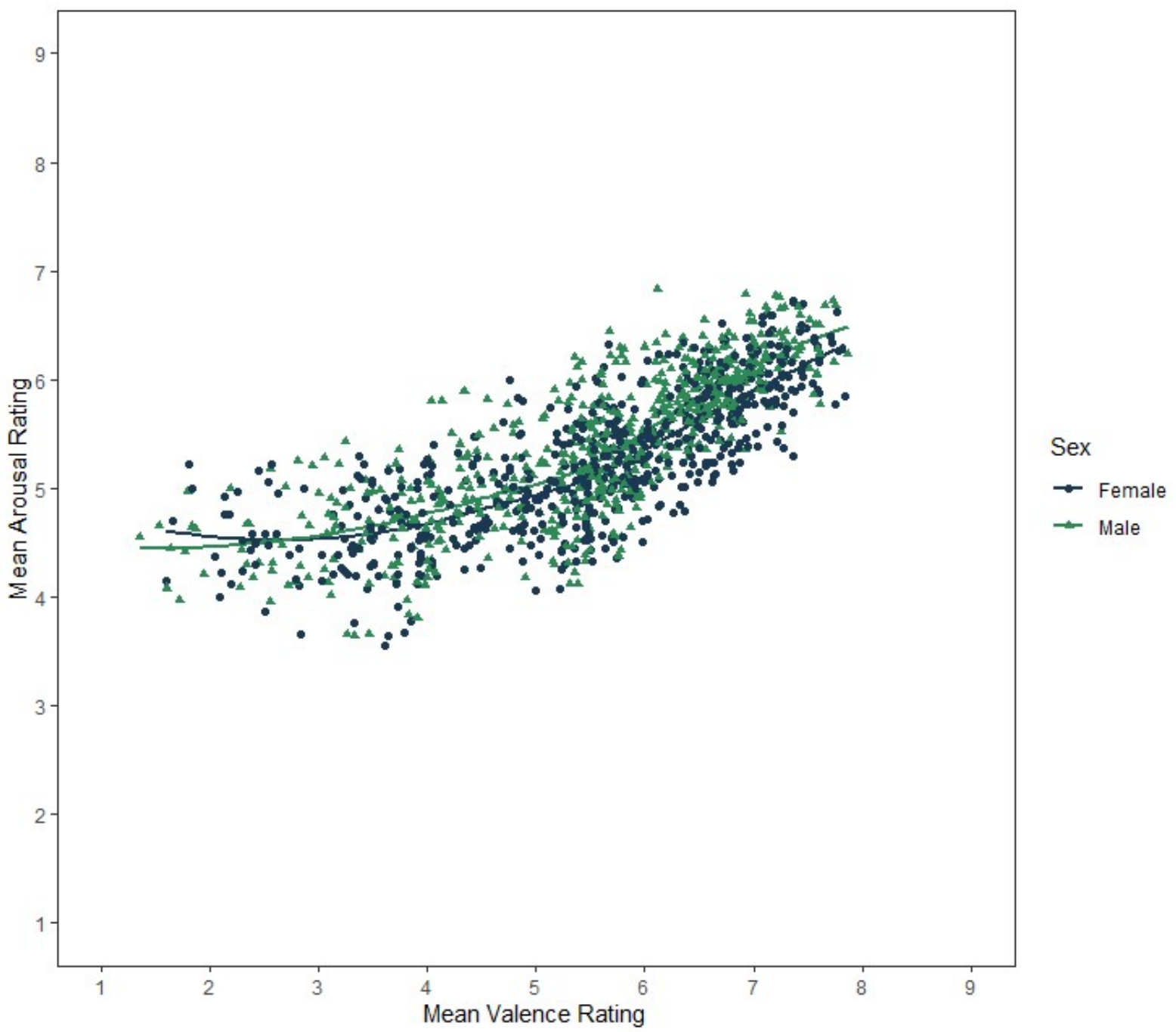


Figure 6

369 Mean Valence by Mean Arousal Ratings per Picture by Age Group from Chinese Participants,

370 with Corresponding LOWESS Lines.

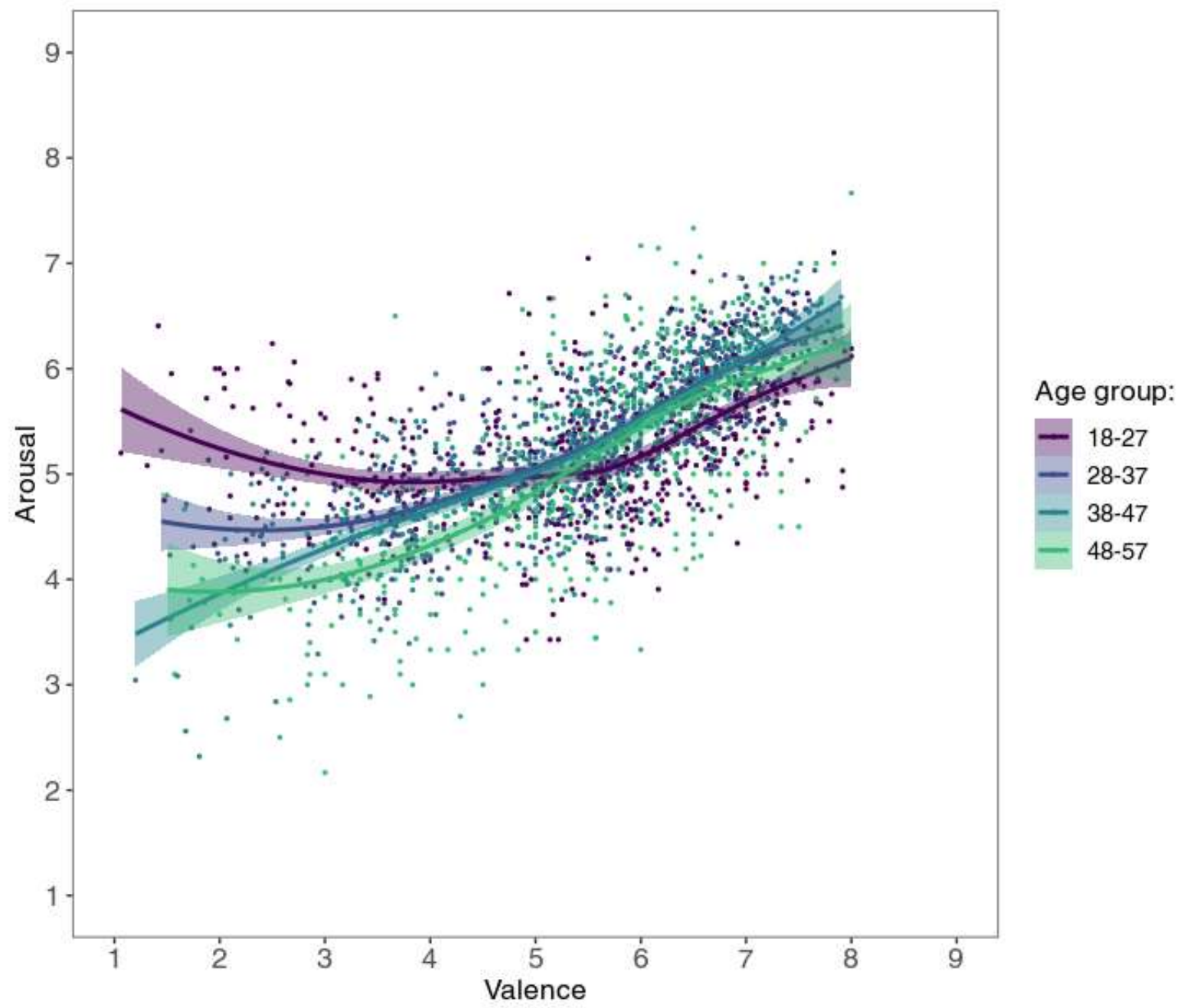

Comparison of US and Chinese Ratings

Mean ratings in the valence category from the US sample showed extremely high

375 positive correlation with those from the Chinese rating, $r(581)=.936,95 \%$ CI $[.925, .946], p$

$376<.001, B F_{10}>10^{100}$ (see Figure 7). A paired $t$-test showed that Americans rated images more 
377 positive than Chinese, with a statistically significant but practically negligible difference of 0.18 ,

378

379 95\% CI $[0.12,0.23]$ (US: 5.64 \pm 1.76 , China: $5.46 \pm 1.37), t(582)=6.35, p<.001, d=0.26,95 \%$ CI $[0.18,0.35], B F_{10}=1.21 \times 10^{7}$. Both these findings are very similar for all age groups and both sexes (see Figure 8; Table A2).

The mean ratings for arousal showed only a weak, albeit significant, correlation, $r(581)$ $=.282,95 \%$ CI $[.206, .355], p<.001, B F_{10}=2.30 \times 10^{9}$ (see Figure 9). The lower correlation in the arousal (as compared to the valence) dimension relates to the fact that, as mentioned above, the data from US raters showed a U-shaped valence-arousal-relationship, but the Chinese average affective ratings displayed a linear relationship. However, just as the ratings by younger age groups showed more visually similar valence-arousal patterns (see Figure 6), they also demonstrated substantially higher correlation with US arousal ratings, as most clearly reflected in the youngest age group (see Figure $10 ; r=.53$ as opposed to $.22, .17$, and -.07 for the other three age groups, see Table A2; $p<.001$ for all $z$-test comparisons; Diedenhofen \& Musch, 2015). This pattern of age group influence is similar for both males and females, though the correlation was generally higher for males ( $r=.39$ vs .20 ; Table A2; see also all figures via osf.io/anej6 with male and female ratings in separate panels).

A paired $t$-test showed that arousal ratings by Americans were lower than those by Chinese, again a statistically significant yet very small difference, $-0.31,95 \%$ CI $[-0.40,-0.23]$ (mean rating US: $5.00 \pm 1.02$, China: $5.31 \pm 0.64), t(582)=-7.30, p<.001, d=-0.30,95 \% \mathrm{CI}$ $[-0.39,-0.22], B F_{10}=4.95 \times 10^{9}$. This relation is similar for all age groups and both sexes (Table A2). 
All in all, we may conclude that the consensus for valence ratings was generally higher

399 than for arousal ratings, both within a sample of a given culture (see correlation tests for

400 interrater reliability, gender, and age) and between the two cultures tested here.

\section{$401 \quad$ Figure 7}

402 Relationship between US and Chinese Mean Valence Ratings (95\% CI Error Bars) per Image,

403 with the LOWESS Line in Blue.

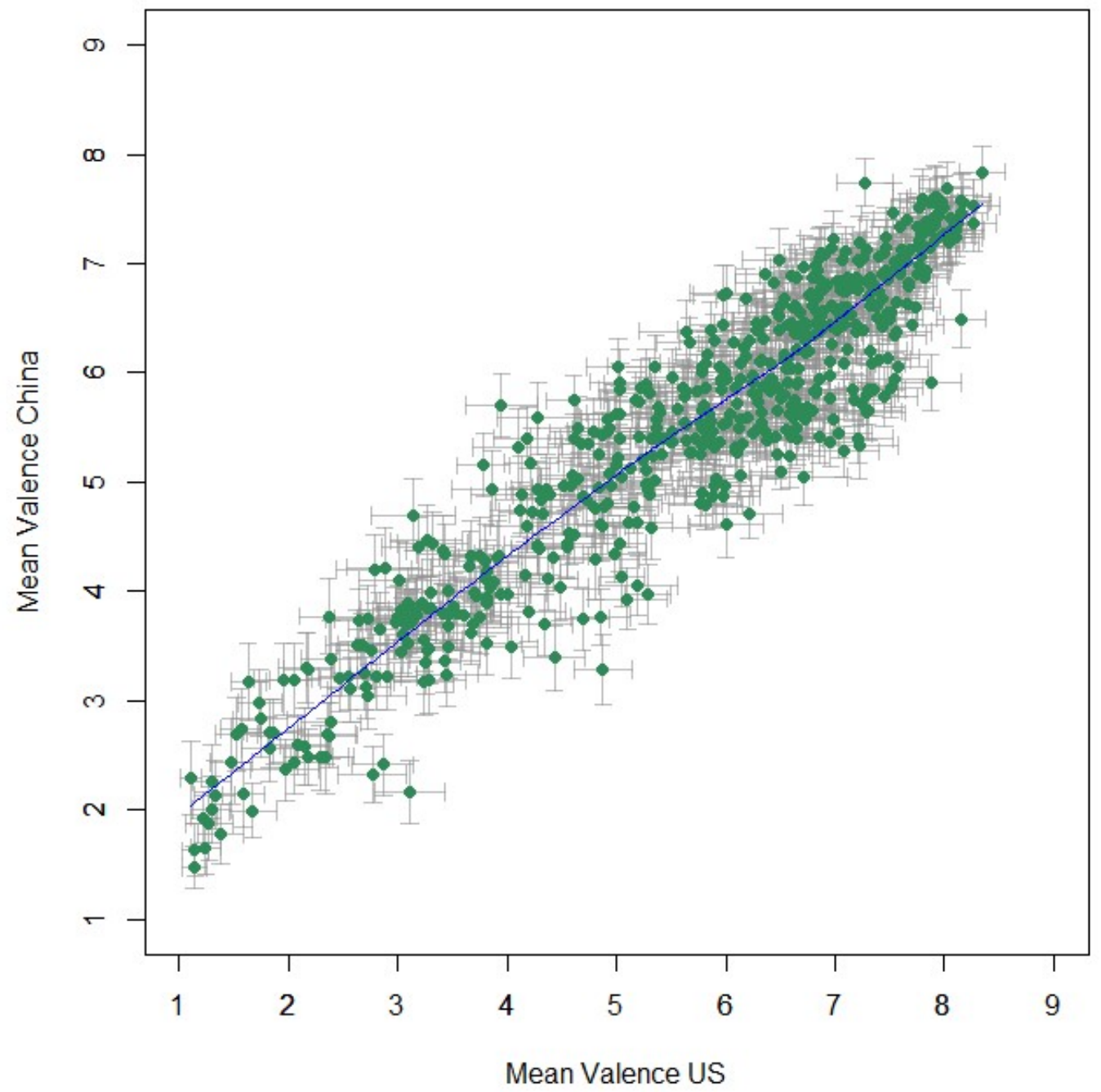


BASS

\section{$404 \quad$ Figure 8}

405 Relationship between US and Chinese Mean Valence Ratings per Image by Age Group, with 406 Corresponding LOWESS Lines.

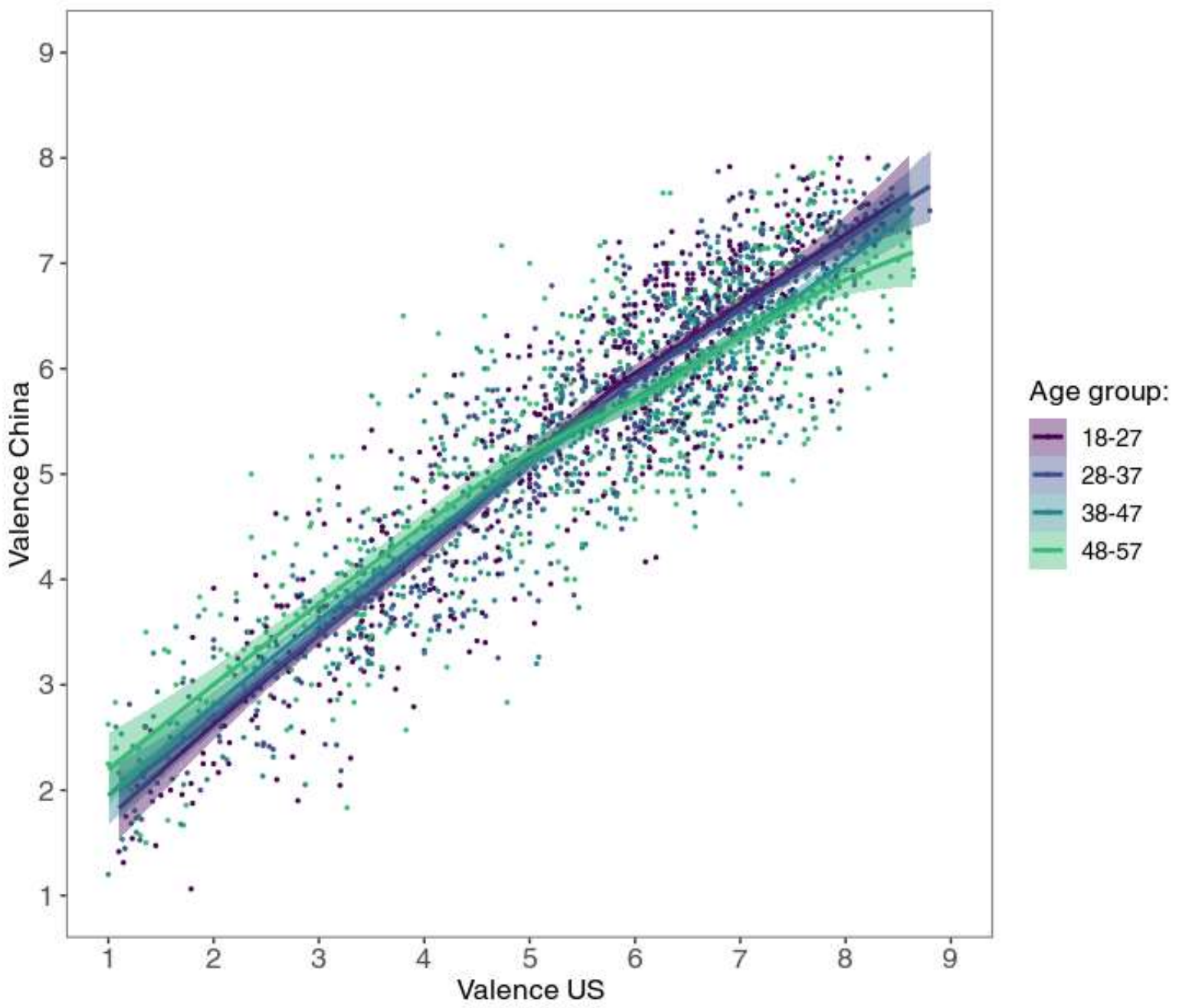


408 Figure 9

409 Relationship between US and Chinese Mean Arousal Ratings (95\% CI Error Bars) per Image,

410 with the LOWESS Line in Blue.

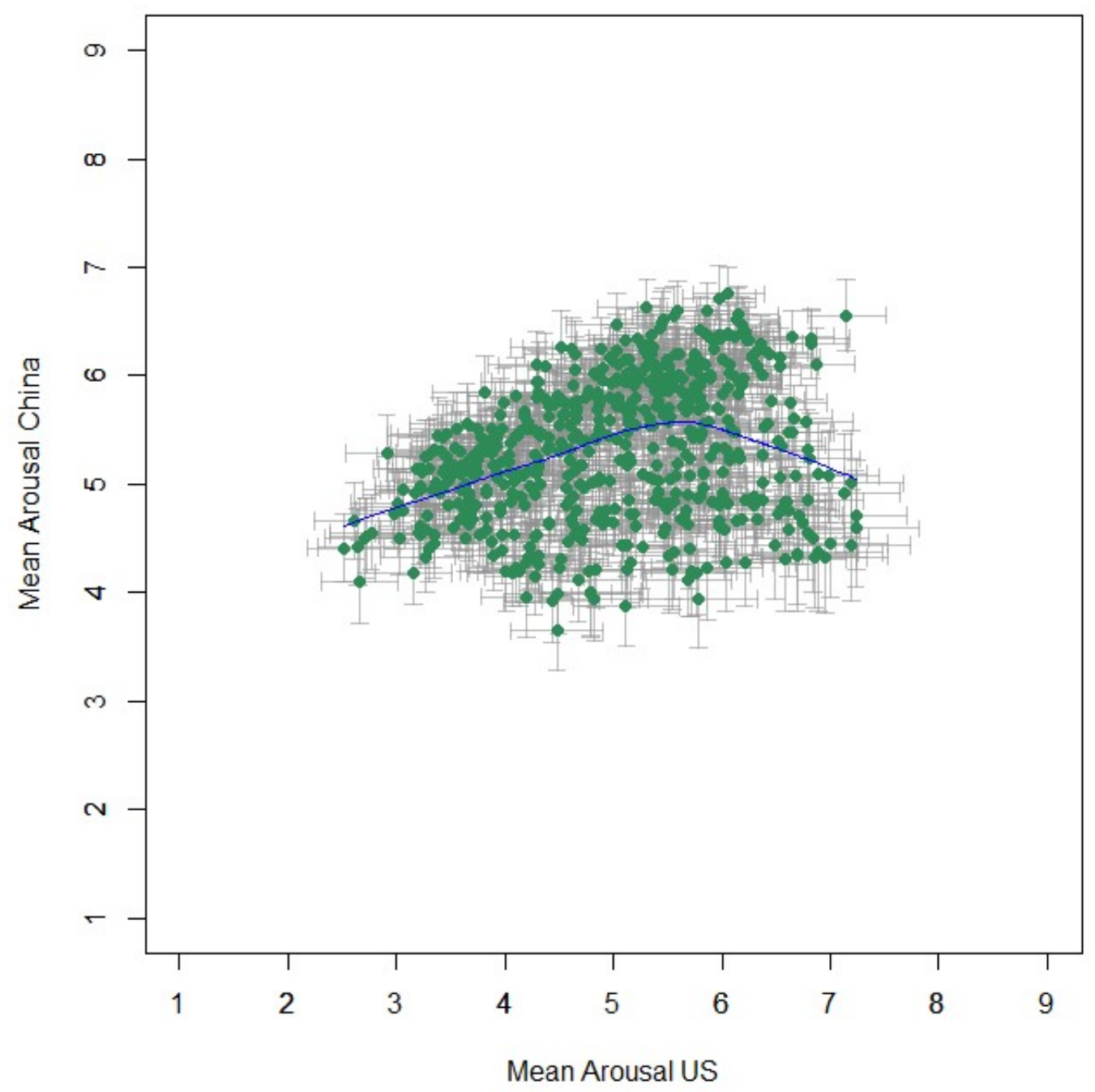

411 
BASS

\section{$415 \quad$ Figure 10}

416 Relationship between US and Chinese Mean Arousal Ratings per Image by Age Group, with

417 Corresponding LOWESS Lines.

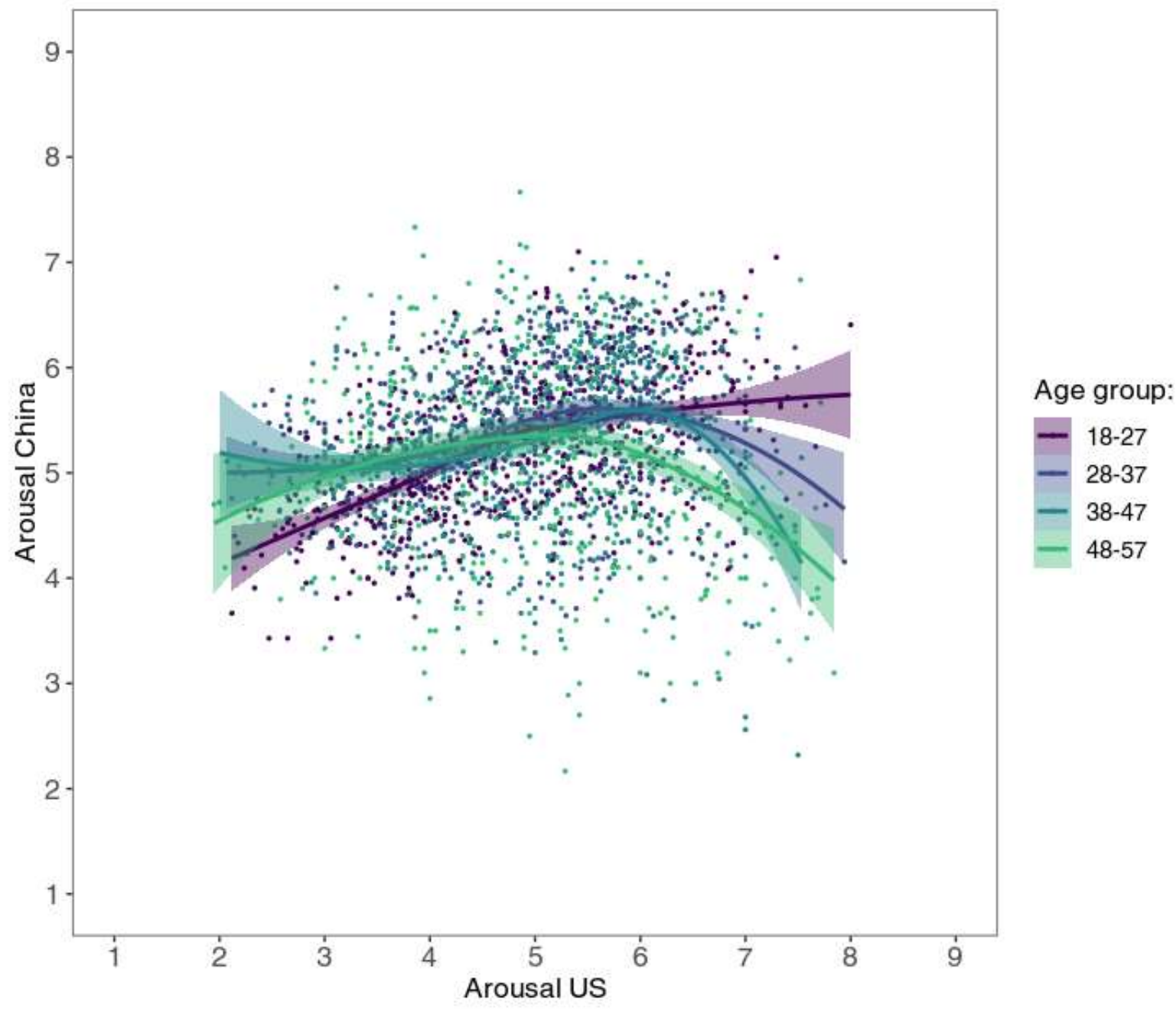

418 


\section{Example Experiment: Color-Valence Congruence Effects in the BASS}

We also included an example experiment, in which we tested the suitability of the BASS images for online research. Here, we manipulated color to demonstrate one major advantage of the BASS images: their easy rendition in a different color. Content-wise, it has been repeatedly shown that the stimulus valence of words interacts with perceptual features like color and brightness: Positive stimuli are associated with green (rather than red) and with white (rather than black; Kawai et al., 2020; Kuhbandner \& Pekrun, 2013; Lakens et al., 2012; Meier et al., 2004, 2015; Moller et al., 2009). As a validation of the suitability of the BASS for online research, in a web-based fast decision task, we tested if the BASS silhouettes can induce similar effects. Details about the experiment can be found in the online supplementary material. Below we provide only a brief summary.

Method. We selected a subset of 60 positive and 60 negative silhouettes from the BASS (a list of filenames is provided in Table C1 in Appendix C). These 120 images were presented to 90 students (age $=23.0 \pm 3.5 ; 27$ male) from the University of Vienna in two experimental blocks: a "color" block, containing each silhouette once in red and once in green color; and a "brightness" block, containing each silhouette once in black and once in white (with silhouette background always in gray, see Figure $\mathrm{C} 1$ for examples). The task was to categorize the valence of each silhouette via key press as either positive or negative. For each block, we ran a repeatedmeasures analysis of variance (ANOVA) on the correct mean response times (RTs) and another on the mean error rates (ERs).

Results. According to expectations, the two (Color: red vs. green; within) $\times$ two (Valence: positive vs. negative; within) ANOVA in the color block showed a significant interaction between color and valence for RTs, $F(1,89)=91.46, p<.001, \eta_{\mathrm{p}}^{2}=.507,90 \% \mathrm{CI}$ 
$443[.383, .595], \eta_{\mathrm{G}}^{2}=.022, B F_{10}=3.10 \times 10^{9}$, and also for ERs, $F(1,89)=83.67, p<.001, \eta_{\mathrm{p}}^{2}$

$444=.485,90 \% \mathrm{CI}[.359, .576], \eta_{\mathrm{G}}^{2}=.106, B F_{10}=2.30 \times 10^{15}$. Positive silhouettes were categorized

445 faster and more accurately when presented in green rather than red, and, inversely, negative

446 silhouettes were categorized faster and more accurately when presented in red rather than green.

447 Also, in the brightness block, the silhouettes elicited the expected brightness-valence interactions

448 in both RTs, $F(1,89)=18.68, p<.001, \eta_{\mathrm{p}}^{2}=.173,90 \%$ CI $[.068, .286], \eta_{\mathrm{G}}^{2}=.005, B F_{10}=65.74$,

449 and ERs, $F(1,89)=8.76, p=.004, \eta_{\mathrm{p}}^{2}=.090,90 \% \mathrm{CI}[.017, .191], \eta_{\mathrm{G}}^{2}=.011, B F_{10}=7.55$.

450 Positive silhouettes were categorized faster and more accurately when presented in white rather

451 than black, and inversely, negative silhouettes were categorized faster and more accurately when

452 presented in black rather than white.

453 Means and SDs for RTs and ERs are illustrated in Figures 11 and 12, and the means can

454 be found in Table C2.

455

456

457

458

459

460

461

462

463

464

465 
BASS

466

Figure 11

467 Mean Reaction Times for Positive and Negative Silhouettes in the Color Block (Left) and the 468 Brightness Block (Right). Error Bars Indicate SEMs.

469

470
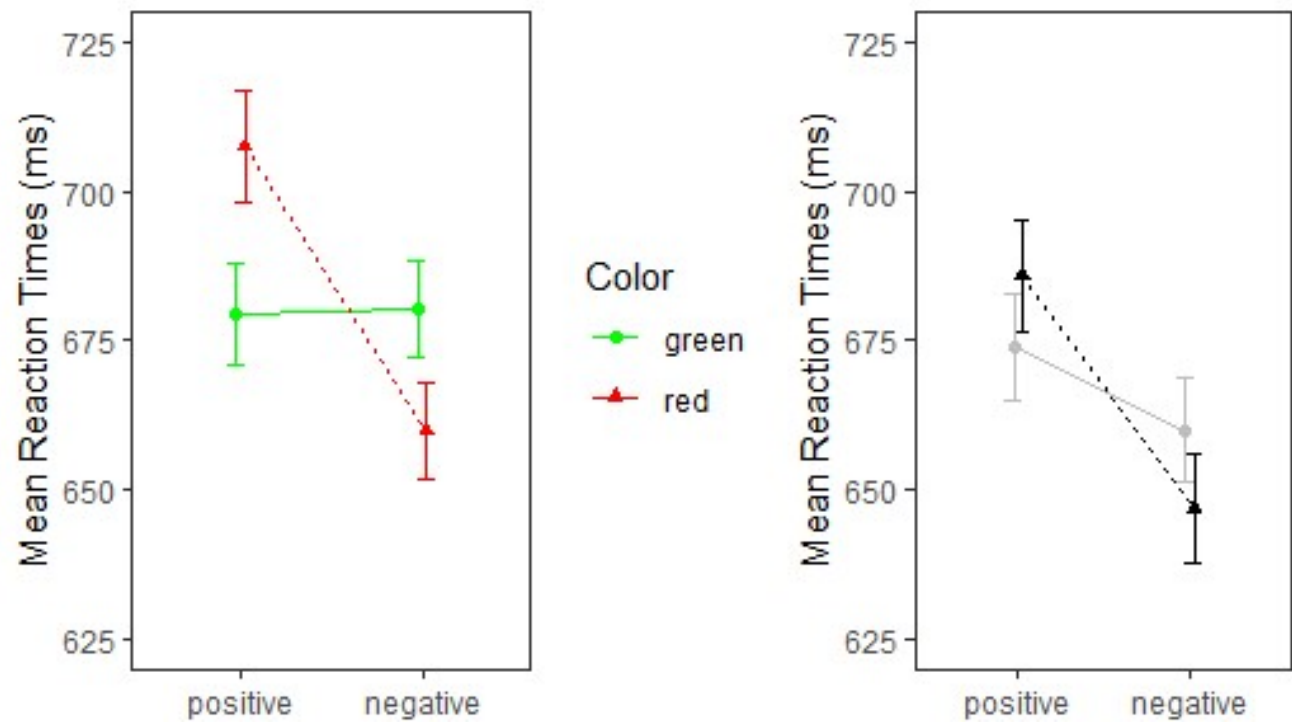

Brightness

Color $\rightarrow$ green

- red

$\rightarrow$ white

- black

\section{$471 \quad$ Figure 12}

472 Mean Error Rates for Positive and Negative Silhouettes in the Color Block (Left) and the

473 Brightness Block (Right). Error Bars Indicate the SEMs.
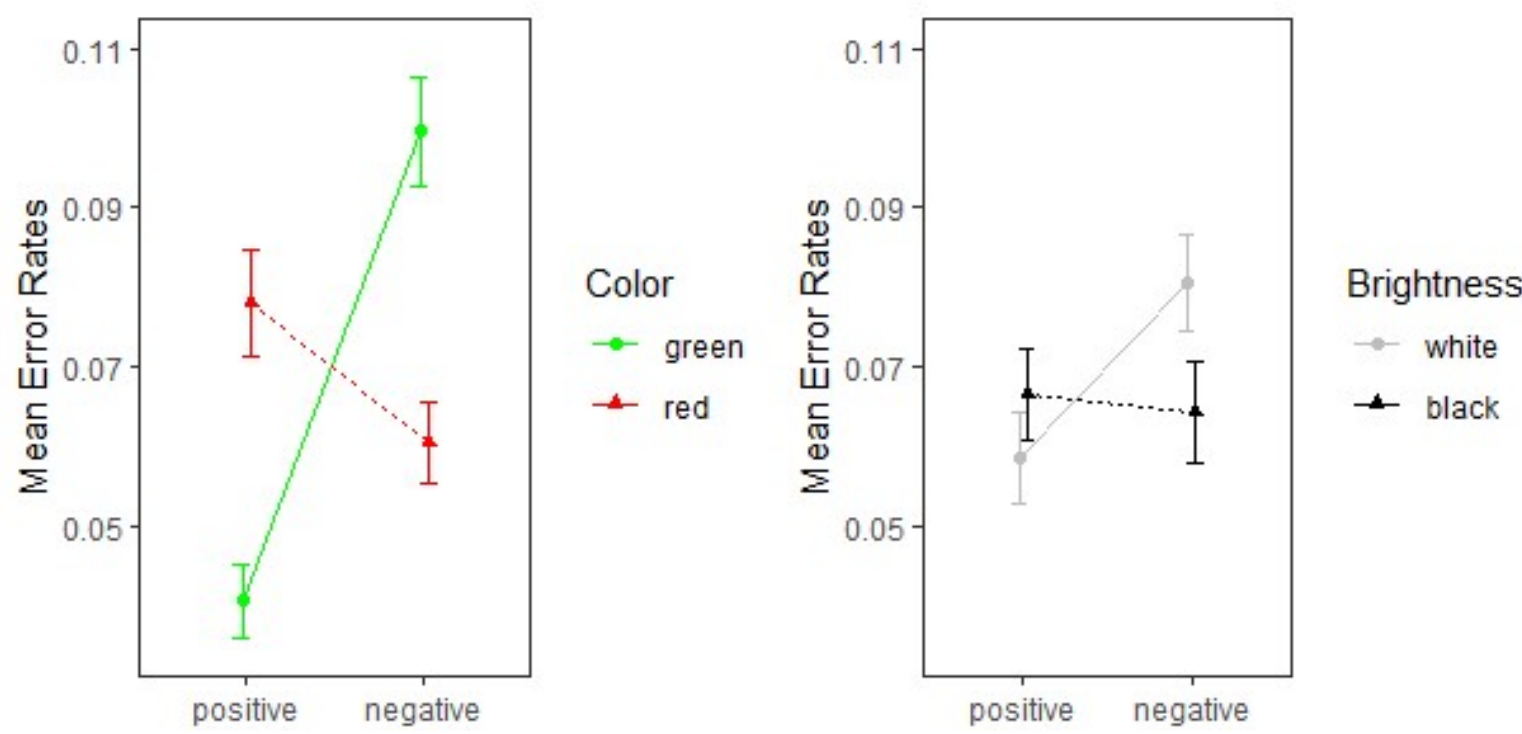
474

475

476

477

478

479

480

481

482

483

484

485

486

487

488

489

490

491

492

493

494

495

496

Discussion

For the current study, we created an affective silhouette database with the specific goal of providing an easy and openly accessible lightweight stimulus pool capable of reliable elicitation of affective representations on a wide range of valence and arousal levels that was validated by extensive samples of two of the biggest culture groups studied in psychology. In the US sample, we found that the images cover a very wide range of affective representations from very negative to very positive (valence), and from very calm to very intense (arousal), indicating various potential applications (see Introduction). The Chinese sample was remarkably similar in valence ratings, covering a wide range with an extremely high correlation with the US sample.

However, the Chinese arousal ratings were only weakly correlated, and comparatively limited in range. This relates to the fact that data from the US participants demonstrated a U-shaped relationship between valence and arousal, while data from the Chinese participants, instead, displayed a more linear valence-arousal relationship (strong positive correlation between valence and arousal ratings) on average.

Studies that look at affective ratings for emotional words in Chinese find a U-shaped valence-arousal relation similar to the one apparent in the US ratings (Ho et al., 2015; Liu et al., 2018; Yao et al., 2017). It is noteworthy, that participants in the affective word studies were high school or university students. Our Chinese sample was more diverse: The age-group-wise analysis shows that younger Chinese display a more quadratic relationship which becomes more linear with increasing age. This provides a plausible reconciliation of these previous findings with our results in that it is only the relatively older Chinese generations whose perception substantially differs from those in Western populations, while the younger generations perceive images more in accordance with the Western population. We can only speculate about the reasons, but the more 
497

498

499

500

501

502

503

504

505

506

507

508

509

510

similar ratings of young US and Chinese participants could reflect a larger shared sphere of visual experiences, for instance, through digital social media (e.g., Tik Tok).

However, two studies that compared affective ratings for emotional photographs (a subset of the IAPS) between Chinese and Western samples interestingly seem to indicate the opposite phenomenon: Gong and Wang (2016) found a high correlation of valence and arousal ratings between Chinese and German older adults ${ }^{12}$, and Huang et al. (2015) argued that Chinese young adults display a different rating behavior from US young adults for IAPS pictures.

Generally, more empirical data on cultural differences in emotion representation or judgments is necessary to establish how and when Chinese rating behavior differs from that of a Western culture. One possibility might be, for instance, that the lower arousal for negative affective silhouettes, which explains most of the difference in our BASS ratings (and the lack of U-shape among Chinese participants, especially with increasing age), might be a result of conflict avoidance or emotion control as a more generally accepted strategy among Chinese than Westerners (Gong \& Wang, 2016; Tjosvold \& Sun, 2002).

We also demonstrated how the BASS images could be used in online experiments. In our example experiment, we showed the typical color-congruence effects of stimulus color on speed and accuracy of judgments about image valences: Positive images were rated faster and more accurately if presented in green than in red, and if presented in white than in black, while the

${ }^{12}$ Gong and Wang (2016) compared their Chinese participant sample with a mean age of $67.3 \pm 4.96$ years to a German participant sample with a mean age of $69.61 \pm 3.58$ years (Grühn \& Scheibe, 2008). Thus, in this comparison, participants were older than our oldest Chinese age group (58+) which had a mean age of $62.36 \pm 5.70$ years and included only 14 participants. 
opposite held true of negative images. ${ }^{13}$ This result replicated prior research and already proves

516 at least one particular use of the BASS images in scientific research.

All in all, the quality of our samples' data (as measured by the respective interrater correlation) is outstanding and, where comparable, its general characteristics are in line with effects observed in notable affective databases like IAPS, OASIS, ANEW and BAWL-R, to name a few.

The BASS, however, aims only to complement, and not to replace, realistic picture databases, for research for which realistic images do not provide ideal stimuli for a given experiment for any of the reasons detailed in the Introduction (e.g., complications of color manipulation, file size, etc.).

While the simplicity of silhouettes can be advantageous for some research designs, their innate lack of context information may simultaneously lead to more (or less) ambiguity in their interpretations between participants than is the case for pictures that are rich in detail. content evaluation if contexts interact with objects in photorealistic image sets, but not or at least

530 less so with silhouettes where context is sparse. However, while the direct interpretation of some 531 of the silhouettes may, thus, be more or less ambiguous than that of a photographic image of the 532 corresponding content (e.g., some participants may perceive a coyote's silhouette simply as a

${ }^{13}$ The finding may seem to contradict the pilot study results showing that black-on-white and (inverted) white-on-black images are rated very similarly, demonstrating equal recognizability. However, in the pilot study, each participant was presented with only one image type (either all images black-onwhite, or all white-on-black). Color-valence congruence effects can depend on dichotomous decision alternatives within the same task (cf. Kawai et al., 2020; Lakens et al., 2012), and, the present results are in line with this possibility and previous research showing that such congruence effects were only elicited in the example experiment (where each task contained contrasting color types alternating trial by trial), not during the affective rating study. 
533 dog when presented without a fitting habitat as context), in the current study, the affective ratings

534 of the silhouettes generally demonstrated very high consistencies (very high inter-rater

535 correlation, low $S D$ s, and, in case of valence, very high intercultural correlation), which attest to

536 the silhouettes' emotional unambiguousness. Furthermore, several numeric measures in our

537 BASS database, such as the rating differences between US and China per image, the

538 corresponding $B F$ s or residuals, or simply the $S D$ s of the ratings in either sample, allow

539 researchers to choose images of emotional clarity or culture-neutrality suiting their needs best.

540 Nonetheless, and although the rating data of our BASS images correspond well with the

541 data obtained for photographic databases, we cannot rule out that the silhouettes evoke smaller

542 emotional responses than contextually richer photographic pictures when using other measures

543 (e.g., skin conductance, heart rate, facial muscles activities). Do photorealistic pictures elicit

544 stronger emotion-related physiological responses than silhouettes, and if so, what are the driving

545 forces in such a case (object detail, color, depth and perspective, etc.)? We hope that future

546 research, facilitated by the BASS, will help to address these questions.

\section{Conclusion}

The BASS offers a novel database of visual silhouettes for psychological research

550 optimally suited for the study of image-elicited affective representations in online experiments

551 and validated by normative ratings by representative Western and Asian samples.

\section{Declarations}

$554 \quad$ Funding 

number 25068, G.L.'s personal grant number 24945).

\section{Conflicts of interest}

The authors have no competing interests.

\section{Ethics, consent to participate, and consent for publication}

The research was conducted according to the principles expressed in the Declaration of Helsinki. During online participation, no potentially identifying information such as IP addresses was collected and participants were informed that (i) their data would be treated anonymously, and (ii) they could unconditionally stop their participation. Participants were informed that their anonymized data would be published and were given the opportunity to prohibit the use of their data. Informed consent was obtained from all subjects.

\section{Availability of data, materials, and code}

The BASS and its accompanying data table are available for download from the OSF project page under osf.io/anej6. The project page further contains all preregistrations, collected raw data, experimental and analysis scripts, a useful R-script to convert the colors of the silhouettes, as well as detailed reports on the procedures involved in the creation of the BASS and its follow-up experiment. A browsable and downloadable version of the complete database (silhouette images and data table) is also available via https:/gasparl.github.io/BASS/.

\section{Authors' contributions}

Concept by C.K. and U.A., images by C.K., study design and analysis by C.K. and G.L., presentation software by G.L. and C.K., manuscript by C.K., G.L., and U.A. 


\section{Acknowledgments}

Claudia Kawai and Gáspár Lukács are recipients of DOC Fellowships of the Austrian

578 Academy of Sciences at the Department of Cognition, Emotion, and Methods in Psychology at

579 the University of Vienna. The research was further supported by Dr. Franziska Pinsker, for

580 which we thank her. We want to acknowledge the assistance of Yang Zhang, who translated the

581 experiment to Mandarin and collected the online pilot data in China. We also thank Wenyi Chu

582 for her feedback on clarity of the experimental procedure, and Sophie Hanke, Tobias Greif, and

583 Bence Szaszkó for making browsing the BASS easier. The meticulous comments Anna Walker

584 provided are greatly appreciated. Special thanks goes to the talented Anna Lumaca

585 (allhailthesnail.com) for creating and contributing 20 much needed "ghastly" silhouettes to the 586 BASS. 


\section{References}

Anwyl-Irvine, A., Dalmaijer, E. S., Hodges, N., \& Evershed, J. K. (2020). Realistic precision and accuracy of online experiment platforms, web browsers, and devices. Behavior Research Methods. https://doi.org/10.3758/s13428-020-01501-5

Aro, M., \& Wimmer, H. (2003). Learning to read: English in comparison to six more regular orthographies. Applied Psycholinguistics, 24(4), 621-635. https://doi.org/10.1017/S0142716403000316

Baker, A., Mystkowski, J., Culver, N., Yi, R., Mortazavi, A., \& Craske, M. G. (2010). Does habituation matter? Emotional processing theory and exposure therapy for acrophobia. Behaviour Research and Therapy, 48(11), 1139-1143. https://doi.org/10.1016/j.brat.2010.07.009

Bar, M. (2007). The proactive brain: Using analogies and associations to generate predictions. Trends in Cognitive Sciences, 11(7), 280-289. https://doi.org/10.1016/j.tics.2007.05.005

Biederman, I. (1987). Recognition-by-components: A theory of human image understanding. Psychological Review, 94, 115-147. https://doi.org/10.1037/0033-295X.94.2.115

Brady, T. F., Konkle, T., Gill, J., Oliva, A., \& Alvarez, G. A. (2013). Visual long-term memory has the same limit on fidelity as visual working memory. Psychological Science, 24(6), 981-990. https://doi.org/10.1177/0956797612465439

Bradley, M. M., Codispoti, M., Sabatinelli, D., \& Lang, P. J. (2001). Emotion and motivation II: Sex differences in picture processing. Emotion, 1(3), 300-319. https://doi.org/10.1037/1528-3542.1.3.300 
609

610

611

612

613

614

615

616

617

618

619

620

621

622

623

624

625

626

627

628

629

630

Bradley, M. M., \& Lang, P. J. (2007). The International Affective Picture System (IAPS) in the study of emotion and attention. In Handbook of emotion elicitation and assessment (pp. 29-46). Oxford University Press.

Dan-Glauser, E. S., \& Scherer, K. R. (2011). The Geneva affective picture database (GAPED): A new 730-picture database focusing on valence and normative significance. Behavior Research Methods, 43(2), 468-477. https://doi.org/10.3758/s13428-011-0064-1

Deák, A., Csenki, L., \& Révész, G. (2010). Hungarian ratings for the International Affective Picture System (IAPS): A cross-cultural comparison. Empirical Text and Culture Research, 4(8), 90-101.

Diedenhofen, B., \& Musch, J. (2015). cocor: A Comprehensive Solution for the Statistical Comparison of Correlations. PLOS ONE, 10(4), Article e0121945. http://dx.doi.org/10.1371/journal.pone.0121945

Duñabeitia, J. A., Crepaldi, D., Meyer, A. S., New, B., Pliatsikas, C., Smolka, E., \& Brysbaert, M. (2018). MultiPic: A standardized set of 750 drawings with norms for six European languages. Quarterly Journal of Experimental Psychology, 71(4), 808-816. https://doi.org/10.1080/17470218.2017.1310261

Fazio, R. H. (2001). On the automatic activation of associated evaluations: An overview. Cognition \& Emotion, 15(2), 115-141. https://doi.org/10.1080/0269993004200024

Foa, E. B., \& Kozak, M. J. (1986). Emotional processing of fear: Exposure to corrective information. Psychological Bulletin, 99(1), 20-35. https://doi.org/10.1037/00332909.99.1.20 
631 Frost, R., Katz, L., \& Bentin, S. (1987). Strategies for visual word recognition and

632

633

634

635

636

637

638

639

640

641

642

643

644

645

646

647

648

649

650

651

652 orthographical depth: A multilingual comparison. Journal of Experimental Psychology:

Human Perception and Performance, 13(1), 104-115. https://doi.org/10.1037/00961523.13.1.104

Garaizar, P., \& Reips, U.-D. (2019). Best practices: Two web-browser-based methods for stimulus presentation in behavioral experiments with high-resolution timing requirements. Behavior Research Methods, 51(3), 1441-1453. https://doi.org/10.3758/s13428-018-1126-4

Geusebroek, J.-M., Burghouts, G. J., \& Smeulders, A. W. M. (2005). The Amsterdam Library of Object Images. International Journal of Computer Vision, 61(1), 103-112. https://doi.org/10.1023/B:VISI.0000042993.50813.60

Gong, X., \& Wang, D. (2016). Applicability of the International Affective Picture System in Chinese older adults: A validation study: Cross-cultural validity of the IAPS. PsyCh Journal, 5(2), 117-124. https://doi.org/10.1002/pchj.131

Grühn, D., \& Scheibe, S. (2008). Age-related differences in valence and arousal ratings of pictures from the International Affective Picture System (IAPS): Do ratings become more extreme with age? Behavior Research Methods, 40(2), 512-521. https://doi.org/10.3758/BRM.40.2.512

Hansen, T., \& Gegenfurtner, K. R. (2009). Independence of color and luminance edges in natural scenes. Visual Neuroscience, 26(1), 35-49. https://doi.org/10.1017/S0952523808080796

Hermans, D., De Houwer, J., \& Eelen, P. (2001). A time course analysis of the affective priming effect. Cognition \& Emotion, 15(2), 143-165. https://doi.org/10.1080/02699930125768 
653

654

655

656

657

658

659

660

661

662

663

664

665

666

667

668

669

670

671

672

673

674

675

Ho, S. M. Y., Mak, C. W. Y., Yeung, D., Duan, W., Tang, S., Yeung, J. C., \& Ching, R. (2015). Emotional valence, arousal, and threat ratings of 160 Chinese words among adolescents. PLOS ONE, 10(7), Article e0132294. https://doi.org/10.1371/journal.pone.0132294

Huang, J., Xu, D., Peterson, B. S., Hu, J., Cao, L., Wei, N., Zhang, Y., Xu, W., Xu, Y., \& Hu, S. (2015). Affective reactions differ between Chinese and American healthy young adults: A cross-cultural study using the international affective picture system. BMC Psychiatry, 15(1), Article 60. https://doi.org/10.1186/s12888-015-0442-9

Huston, J. P., Nadal, M., Mora, F., Agnati, L. F., \& Conde, C. J. C. (2015). Art, aesthetics, and the brain. OUP Oxford.

Kawai, C., Lukács, G., \& Ansorge, U. (2020). Polarities influence implicit associations between colour and emotion. Acta Psychologica, 209, 103143. https://doi.org/10.1016/j.actpsy.2020.103143

Kuhbandner, C., \& Pekrun, R. (2013). Joint effects of emotion and color on memory. Emotion, 13(3), 375-379. https://doi.org/10.1037/a0031821

Kurdi, B., Lozano, S., \& Banaji, M. R. (2017). Introducing the Open Affective Standardized Image Set (OASIS). Behavior Research Methods, 49(2), 457-470. https://doi.org/10.3758/s13428-016-0715-3

Lakens, D., Fockenberg, D. A., Lemmens, K. P. H., Ham, J., \& Midden, C. J. H. (2013). Brightness differences influence the evaluation of affective pictures. Cognition $\&$ Emotion, 27(7), 1225-1246. https://doi.org/10.1080/02699931.2013.781501

Lakens, D., Semin, G. R., \& Foroni, F. (2012). But for the bad, there would not be good: Grounding valence in brightness through shared relational structures. Journal of Experimental Psychology: General, 141(3), 584-594. https://doi.org/10.1037/a0026468 
676

677

678

679

680

681

682

683

684

685

686

687

688

689

690

691

692

693

694

695

696

697

698

Lang, P. J., Bradley, M. M., \& Cuthbert, B. N. (1997). International affective picture system (IAPS): Technical manual and affective ratings. NIMH Center for the Study of Emotion and Attention, 1, 39-58.

Lau, R. R., Sears, D. O., \& Jessor, T. (1990). Fact or artifact revisited: Survey instrument effects and pocketbook politics. Political Behavior, 12(3), 217-242. https://doi.org/10.1007/BF00992334

Leder, H., Tinio, P. P. L., \& Bar, M. (2011). Emotional valence modulates the preference for curved objects. Perception, 40(6), 649-655. https://doi.org/10.1068/p6845

Lindsay, G. W. (2020). Attention in psychology, neuroscience, and machine learning. Frontiers in Computational Neuroscience, 14, Article 29. https://doi.org/10.3389/fncom.2020.00029

Liu, P., Li, M., Lu, Q., \& Han, B. (2018). Norms of valence and arousal for 2,076 Chinese 4character words. In K. Hasida \& W. P. Pa (Eds.), Computational Linguistics (Vol. 781, pp. 88-98). Springer Singapore. https://doi.org/10.1007/978-981-10-8438-6_8

Marchewka, A., Żurawski, Ł., Jednoróg, K., \& Grabowska, A. (2014). The Nencki Affective Picture System (NAPS): Introduction to a novel, standardized, wide-range, high-quality, realistic picture database. Behavior Research Methods, 46(2), 596-610. https://doi.org/10.3758/s13428-013-0379-1

Marr, D. (1982). Vision. A computational investigation into the human representation and processing of visual information. W. H. Freeman and Company.

Meier, B. P., Fetterman, A. K., \& Robinson, M. D. (2015). Black and white as valence cues: A large-scale replication effort of Meier, Robinson, and Clore (2004). Social Psychology, 46(3), 174-178. https://doi.org/10.1027/1864-9335/a000236 
699

700

701

702

703

704

705

706

707

708

709

710

711

712

713

714

715

716

717

718

719

720

Meier, B. P., \& Robinson, M. D. (2004). Why the sunny side is up: Associations between affect and vertical position. Psychological Science, 15(4), 243-247. https://doi.org/10.1111/j.0956-7976.2004.00659.x

Meier, B. P., Robinson, M. D., \& Clore, G. L. (2004). Why good guys wear white: Automatic inferences about stimulus valence based on brightness. Psychological Science, 15(2), 8287. https://doi.org/10.1111/j.0963-7214.2004.01502002.x

Moller, A. C., Elliot, A. J., \& Maier, M. A. (2009). Basic hue-meaning associations. Emotion, 9(6), 898-902. https://doi.org/10.1037/a0017811

Mould, D., Mandryk, R. L., \& Li, H. (2012). Emotional response and visual attention to nonphotorealistic images. Computers \& Graphics, 36(6), 658-672. https://doi.org/10.1016/j.cag.2012.03.039

Nishimoto, T., Ueda, T., Miyawaki, K., Une, Y., \& Takahashi, M. (2012). The role of imageryrelated properties in picture naming: A newly standardized set of 360 pictures for Japanese. Behavior Research Methods, 44(4), 934-945. https://doi.org/10.3758/s13428011-0176-7

Oliva, A., \& Schyns, P. G. (2000). Diagnostic colors mediate scene recognition. Cognitive Psychology, 41, 176-210. https://doi.org/10.1006/cogp.1999.0728

Orzan, A., Bousseau, A., Barla, P., \& Thollot, J. (2007). Structure-preserving manipulation of photographs. Proceedings of the 5th International Symposium on Non-Photorealistic Animation and Rendering - NPAR '07, 103. https://doi.org/10.1145/1274871.1274888

Pôrto, W. G., Bertolucci, P. H. F., \& Bueno, O. F. A. (2010). The paradox of age: An analysis of responses by aging Brazilians to International Affective Picture System (IAPS). Revista 
Brasileira de Psiquiatria, 33(1), 10-15. https://doi.org/10.1590/S151644462010005000015

Proverbio, A. M., Adorni, R., Zani, A., \& Trestianu, L. (2009). Sex differences in the brain response to affective scenes with or without humans. Neuropsychologia, 47(12), 2374 2388. https://doi.org/10.1016/j.neuropsychologia.2008.10.030

Rajalingham, R., Issa, E. B., Bashivan, P., Kar, K., Schmidt, K., \& DiCarlo, J. J. (2018). Largescale, high-resolution comparison of the core visual object recognition behavior of humans, monkeys, and state-of-the-art deep artificial neural networks. The Journal of Neuroscience, 38(33), 7255-7269. https://doi.org/10.1523/JNEUROSCI.0388-18.2018

Ramaswami, M. (2014). Network plasticity in adaptive filtering and behavioral habituation. Neuron, 82(6), 1216-1229. https://doi.org/10.1016/j.neuron.2014.04.035

R Core Team (2020). R: A language and environment for statistical computing. R Foundation for Statistical Computing, Vienna, Austria. URL https://www.R-project.org/

Rossion, B., \& Pourtois, G. (2004). Revisiting Snodgrass and Vanderwart's object pictorial set: The role of surface detail in basic-level object recognition. Perception, 33(2), 217-236. https://doi.org/10.1068/p5117

Sabatinelli, D., Flaisch, T., Bradley, M. M., Fitzsimmons, J. R., \& Lang, P. J. (2004). Affective picture perception: Gender differences in visual cortex? NeuroReport, 15(7), 1109-1112. https://doi.org/10.1097/00001756-200405190-00005

Schmalz, X., Beyersmann, E., Cavalli, E., \& Marinus, E. (2016). Unpredictability and complexity of print-to-speech correspondences increase reliance on lexical processes: More evidence for the orthographic depth hypothesis. Journal of Cognitive Psychology, 28(6), 658-672. https://doi.org/10.1080/20445911.2016.1182172 
744 Schupp, H. T., Markus, J., Weike, A. I., \& Hamm, A. O. (2003). Emotional facilitation of sensory processing in the visual cortex. Psychological Science, 14(1), 7-13. https://doi.org/10.1111/1467-9280.01411

Snodgrass, J. G., \& Vanderwart, M. (1980). A standardized set of 260 pictures: Norms for name agreement, image agreement, familiarity, and visual complexity. Journal of Experimental Psychology: Human Learning and Memory, 6(2), 174-215.

The GIMP Development Team. (2019). GIMP. Retrieved from https://www.gimp.org

Verschuere, B., Crombez, G., \& Koster, E. (2001). The International Affective Picture System: A Flemish Validation Study. Psychologica Belgica, 41(4), 205-217. https://doi.org/10.5334/pb.981

Tjosvold, D., \& Sun, H. F. (2002). Understanding conflict avoidance: Relationship, motivations, actions, and consequences. International Journal of Conflict Management, 13(2), 142 164. https://doi.org/10.1108/eb022872

Võ, M. L. H., Conrad, M., Kuchinke, L., Urton, K., Hofmann, M. J., \& Jacobs, A. M. (2009). The Berlin Affective Word List Reloaded (BAWL-R). Behavior Research Methods, 41(2), 534-538. https://doi.org/10.3758/BRM.41.2.534

Wilcox, N., \& Wlezien, C. (1993). The contamination of responses to survey items: Economic perceptions and political judgments. Political Analysis, 5, 181-213. https://doi.org/10.1093/pan/5.1.181

Wilms, L., \& Oberfeld, D. (2018). Color and emotion: Effects of hue, saturation, and brightness. Psychological Research, 82(5), 896-914. https://doi.org/10.1007/s00426-017-0880-8

Wrase, J., Klein, S., Gruesser, S. M., Hermann, D., Flor, H., Mann, K., Braus, D. F., \& Heinz, A. (2003). Gender differences in the processing of standardized emotional visual stimuli in 
humans: A functional magnetic resonance imaging study. Neuroscience Letters, 348(1), 41-45. https://doi.org/10.1016/S0304-3940(03)00565-2

Yao, Z., Wu, J., Zhang, Y., \& Wang, Z. (2017). Norms of valence, arousal, concreteness, familiarity, imageability, and context availability for 1,100 Chinese words. Behavior Research Methods, 49(4), 1374-1385. https://doi.org/10.3758/s13428-016-0793-2

Zhou, H., \& Fishbach, A. (2016). The pitfall of experimenting on the web: How unattended selective attrition leads to surprising (yet false) research conclusions. Journal of Personality and Social Psychology, 111(4), 493-504. https://doi.org/10.1037/pspa0000056 


\section{Appendix A}

\section{Table A1}

Demographics of the Included Participants from the US and Chinese Ratings with

Corresponding Means and SD for Valence and Arousal.

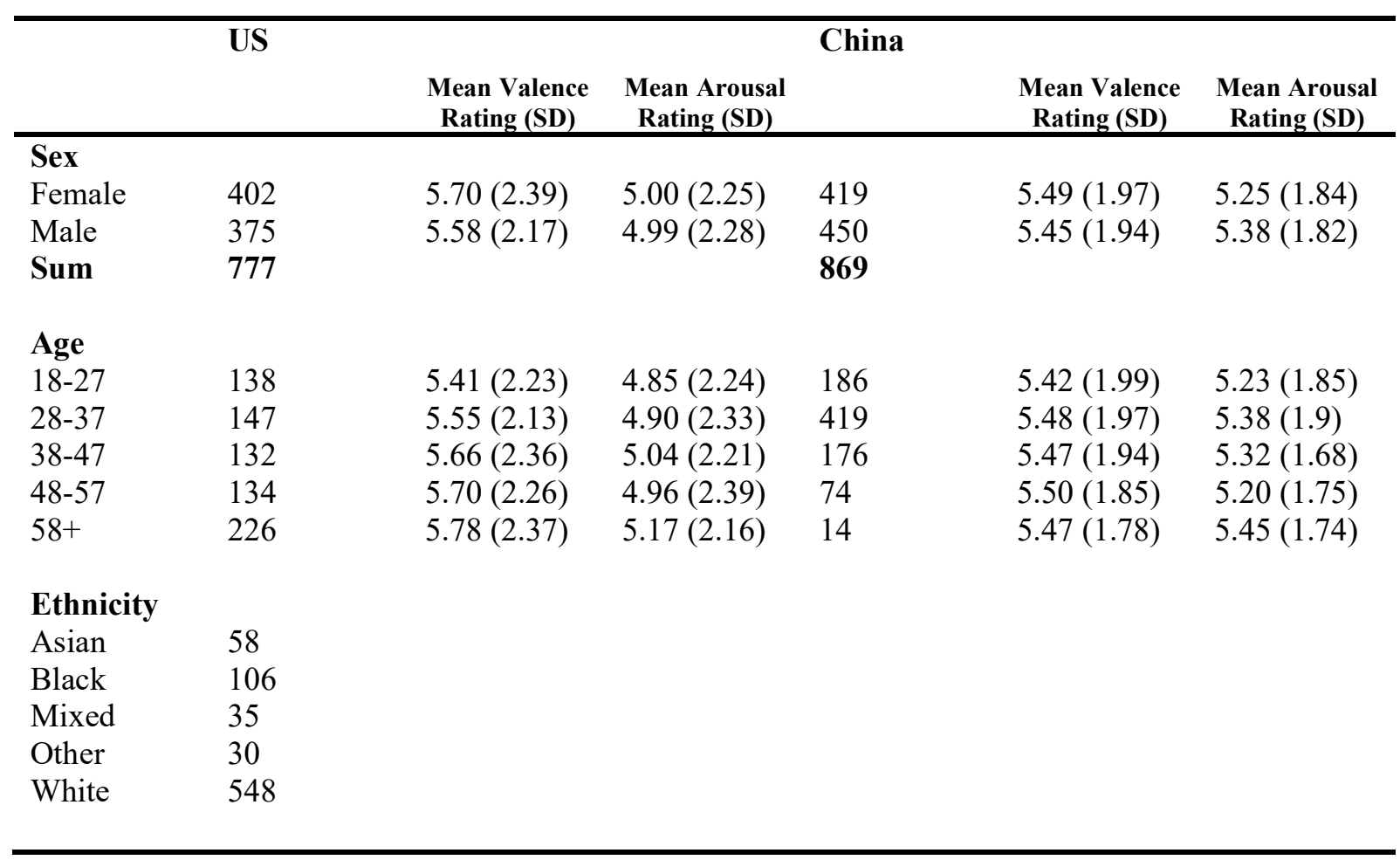


Table A2

US and Chinese Valence and Arousal Rating Correlation per Sex and Age Group.

\begin{tabular}{lll}
\hline & Valence Correlation [95\% CI] & Arousal Correlation [95\% CI] \\
\hline Sex & & $.198[.119, .275]$ \\
Female & $.913[.899, .926]$ & $.387[.316, .454]$ \\
Male & $.942[.933, .951]$ & \\
& & $.530[.469, .586]$ \\
Age & & $.219[.140, .295]$ \\
$18-27$ & $.910[.895, .923]$ & $.170[.090, .248]$ \\
$28-37$ & $.921[.908, .933]$ & $-.069[-.150, .012]$ \\
$38-47$ & $.899[.882, .913]$ & $.251[.173, .326]$ \\
$48-57$ & $.856[.833, .876]$ & \\
$58+*$ & $.689[.644, .730]$ &
\end{tabular}

\footnotetext{
* This oldest age group includes only 14 Chinese participants (cf. Table A1), hence the related correlations are not reliable.
}

\section{Figure A1}

Screenshot of the Experiment's Welcome Page.

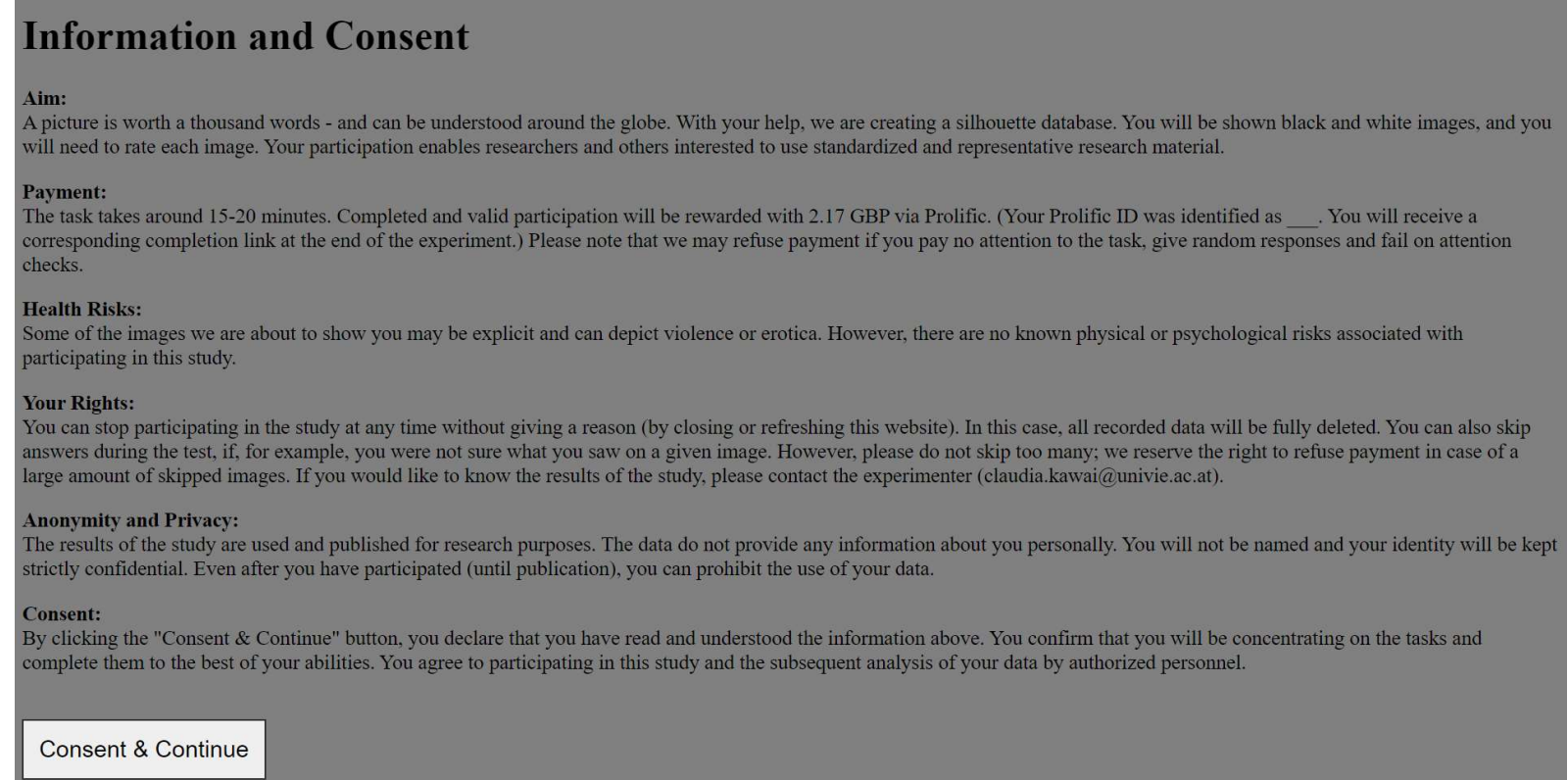




\section{Figure A2}

Screenshot of the Instruction Page After Consenting (Example from the Arousal Substudy).

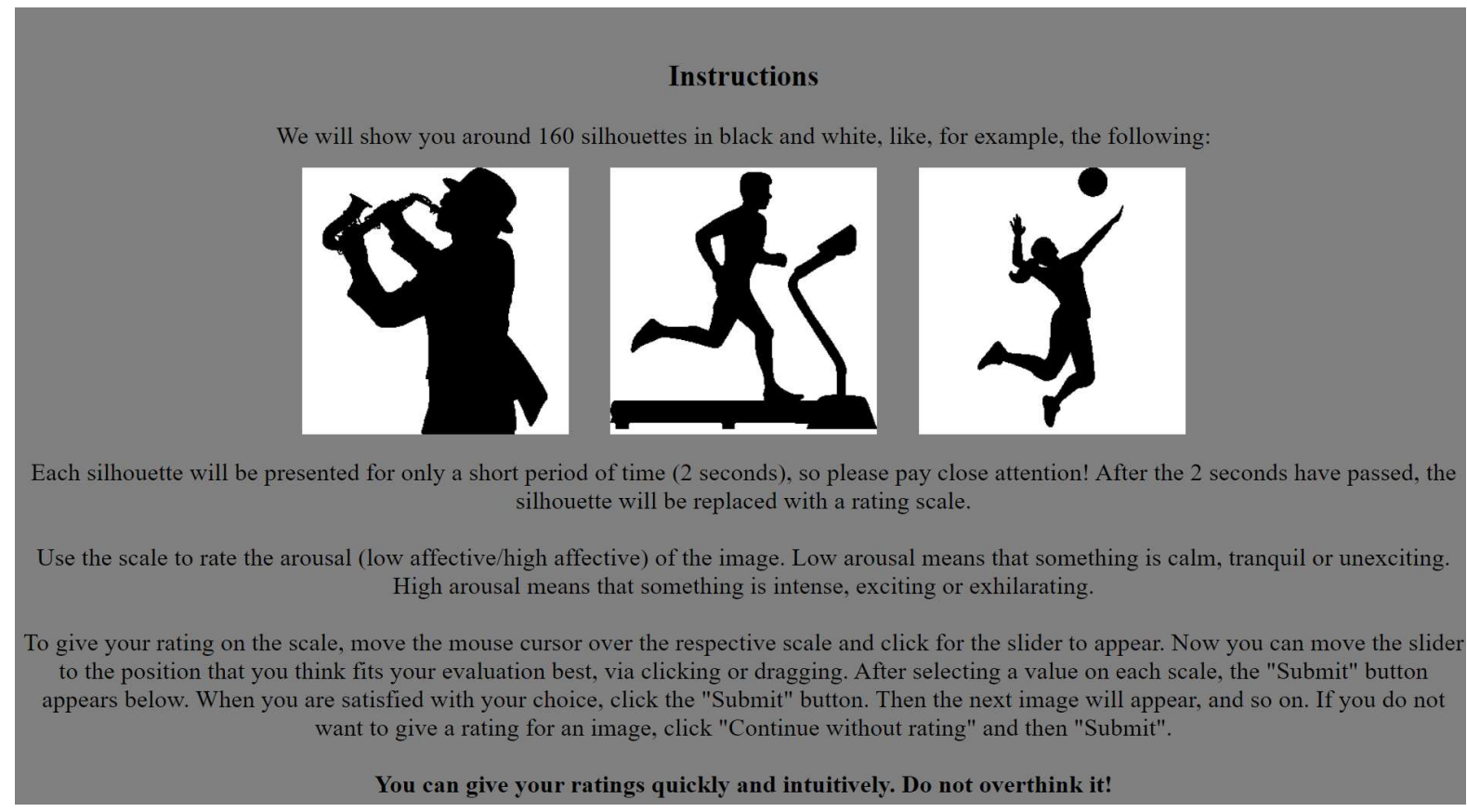

\section{Figure A3}

Screenshot of the Presentation of the Silhouette Stimulus (Start of the Trial), Followed by the Appearance of the Rating Scale (Example from the Arousal Substudy).
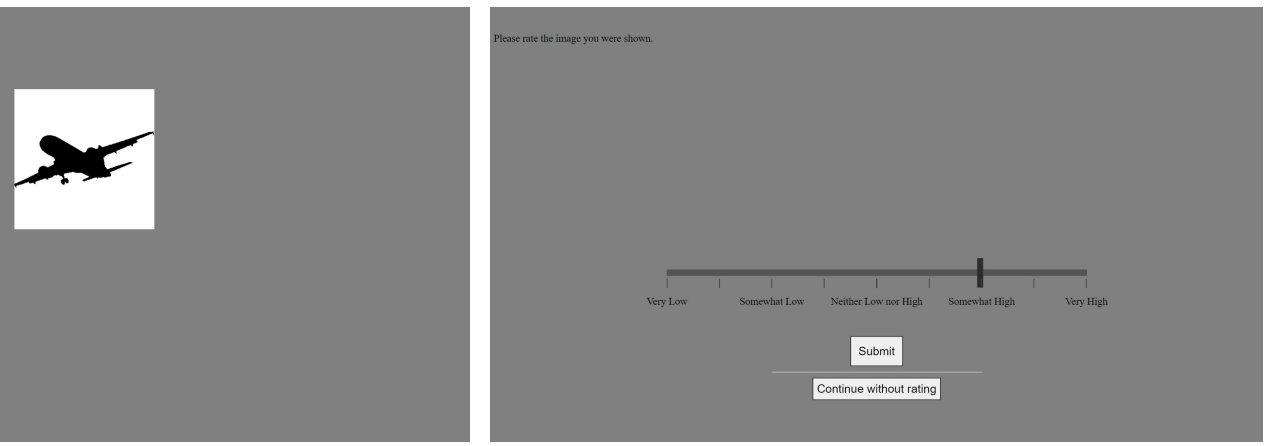


\section{Figure A4}

Mean Valence and Mean Arousal Ratings per Participant According to Their Country, Sex, and Age Group.
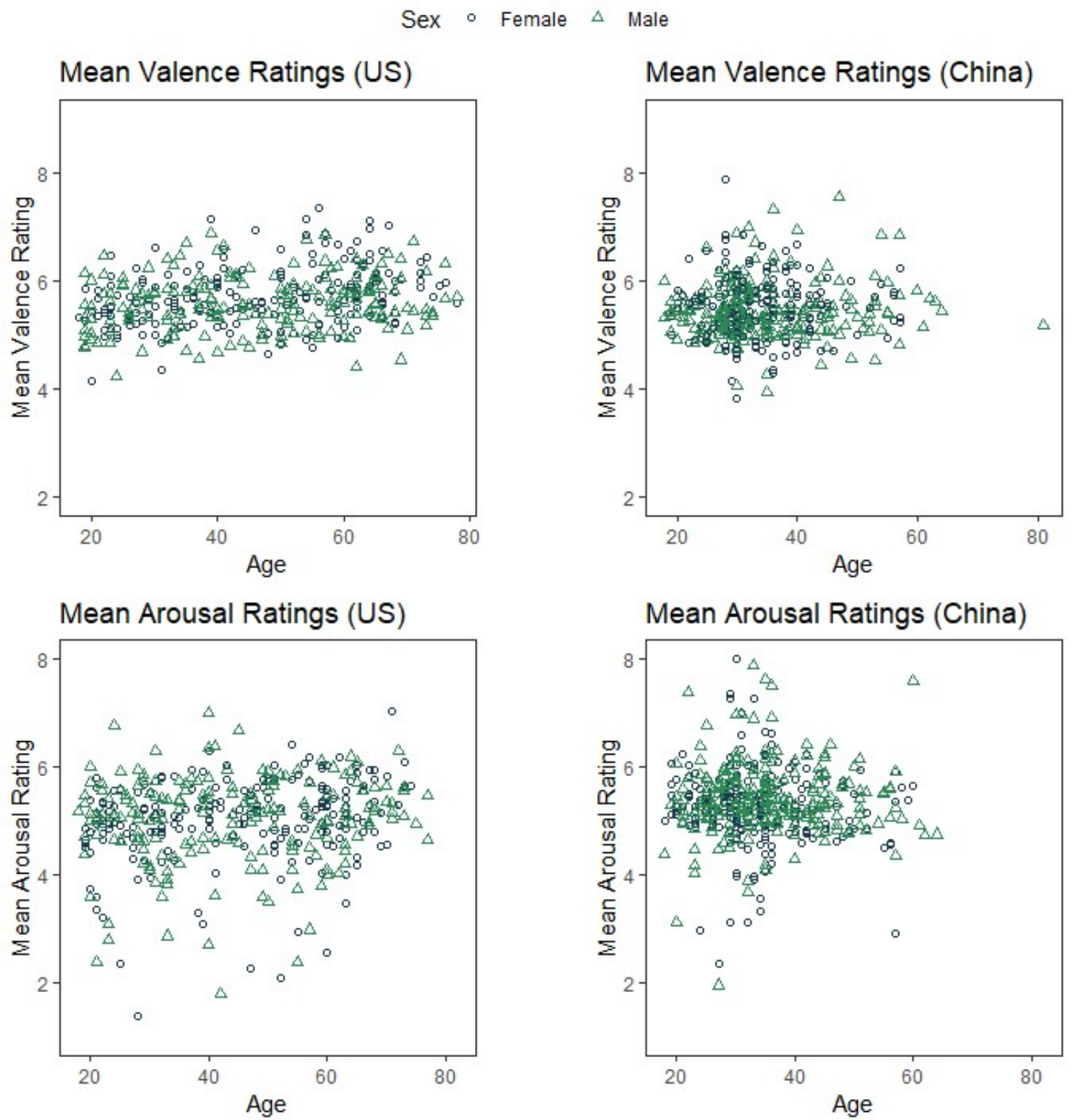


\section{Appendix B}

Table B

Bicolor Affective Silhouette and Shape Database (BASS) Datatable Manual.

\begin{tabular}{|c|c|}
\hline Column & Explanation \\
\hline file_name & The image file name. \\
\hline val_mean_us & The mean of all valence ratings. \\
\hline aro_mean_us & The mean of all arousal ratings. \\
\hline val_sd_us & The SD of all valence ratings. \\
\hline aro_sd_us & The SD of all arousal ratings. \\
\hline val_n_us & The number of valence ratings. \\
\hline aro_n_us & The number of arousal ratings. \\
\hline val_cilo_us & Lower limit of $95 \% \mathrm{CI}$ for the mean of all valence ratings. \\
\hline val_ciup_us & Upper limit of $95 \% \mathrm{CI}$ for the mean of all valence ratings. \\
\hline aro_cilo_us & Lower limit of $95 \% \mathrm{CI}$ for the mean of all arousal ratings. \\
\hline aro_ciup_us & Upper limit of $95 \%$ CI for the mean of all arousal ratings. \\
\hline val_diff & The difference between the means of US and Chinese valence ratings. \\
\hline aro_diff & The difference between the means of US and Chinese arousal ratings. \\
\hline val_bf_10 & Wilcoxon Bayes factor in support of difference between US and Chinese. \\
\hline val_bf_01 & Wilcoxon Bayes factor in support of equivalence between US and Chinese. \\
\hline aro_bf_10 & Wilcoxon Bayes factor in support of difference between US and Chinese. \\
\hline aro_bf_01 & Wilcoxon Bayes factor in support of equivalence between US and Chinese. \\
\hline val_resid & Linear regression residuals for valence ratings: observed values minus fitted. \\
\hline
\end{tabular}




\begin{tabular}{ll}
\hline aro_resid & Linear regression residuals for arousal ratings (same as for valence, see above). \\
black_px & Number of black pixels in the image. \\
white_px & Number of white pixels in the image. \\
keywords & Keywords for the given image. \\
source & Source of the (original) image the silhouette is based on.
\end{tabular}

Note. The columns listed above with "_us" suffixes refer to rating data from the US sample. The data referring to the Chinese ratings is indicated with "_ch" suffixes - here omitted for brevity. (E.g., val_mean_ch: The mean of all valence ratings from China, etc.) 


\section{Appendix C}

\section{Table C1}

Filenames of the Positive and Negative Silhouettes Used in the Verification Study.

\begin{tabular}{llll}
\hline Positive Silhouettes & & Negative Silhouettes & \\
\hline trapeze4 & badminton & injury & handinjury \\
wedding2 & jump & attack2 & grenade \\
love & highjump4 & execution3 & robber \\
singer3 & highjump & abuse2 & bazooka \\
couple6 & acrobat3 & abuse & pollution2 \\
romance & couple5 & hanging & gunman \\
volleyball & skiing2 & drunkard & drunk \\
champion & dance2 & vomit2 & tick \\
euphoria & ferriswheel & argument4 & axe3 \\
iceskater3 & concert & handcuff2 & scorpion2 \\
success2 & iceskater & noose & mosquito \\
affection2 & skateboard & argument3 & gasmask2 \\
cardio & trapeze3 & argument2 & handcuff \\
handball & tennis & dispute & knife2 \\
kiss4 & handball2 & argument6 & pollution \\
ballet & winner & hazard & mosquito2 \\
basketball4 & rockstar & hitman & knife4 \\
propose & carefree & sorrow2 & snake2 \\
kiss3 & basketball & murder2 & wasp \\
joy & breakdance & gunman2 & machete \\
acrobat & skiing3 & beggar2 & tank2 \\
trapeze & soccer & skull2 & soldier2 \\
jump2 & aerobics & scolding2 & gun \\
happiness & highjump2 & gunman3 & kalashnikov \\
surfer2 & snowboard2 & knife & tank \\
skiing & couple7 & axe & wildwest \\
soccer2 & gymnastics3 & accident2 & sniper \\
father2 & roach2 & rifle \\
propose3 & vomit & skull \\
skateboard2 & falling6 & snake \\
\hline
\end{tabular}




\section{Figure C1}

Example for Color-Manipulated Silhouettes (From Left to Right): Original, Red-Gray, Green-Gray,

Black-Gray, and White-Gray (As Used in the Example Experiment).
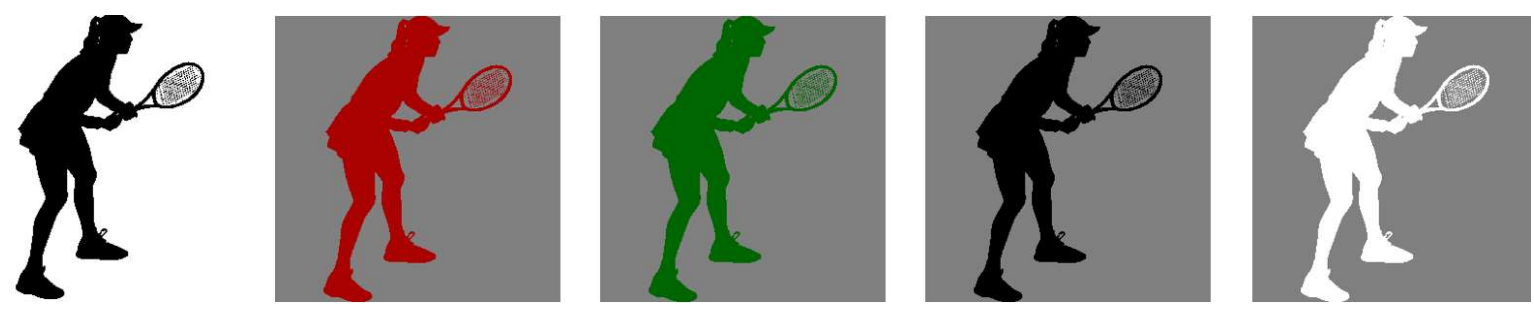

Table C2

Means and SDs for Reaction Times (RTs) and Error Rates (ERs) per Color and Valence Categories in the Bicolor Affective Silhouette and Shape Database (BASS) Validation Experiment.

\begin{tabular}{llll}
\hline Color & Valence & RT $\boldsymbol{M} \pm \boldsymbol{S D}$ & ER $\boldsymbol{M} \pm \boldsymbol{S D}$ \\
\hline Red & Negative & $659.96 \pm 200.61$ & $0.06 \pm 0.24$ \\
Red & Positive & $708.00 \pm 233.20$ & $0.08 \pm 0.27$ \\
Green & Negative & $680.91 \pm 207.14$ & $0.10 \pm 0.30$ \\
Green & Positive & $679.24 \pm 217.30$ & $0.04 \pm 0.20$ \\
Black & Negative & $647.60 \pm 202.37$ & $0.06 \pm 0.25$ \\
Black & Positive & $686.14 \pm 217.78$ & $0.07 \pm 0.25$ \\
White & Negative & $660.16 \pm 202.40$ & $0.08 \pm 0.27$ \\
White & Positive & $674.30 \pm 210.28$ & $0.06 \pm 0.24$ \\
\hline
\end{tabular}

